\title{
Gluon mass generation without seagull divergences
}

\author{
Arlene C. Aguilar ${ }^{1}$ and Joannis Papavassiliou ${ }^{2}$ \\ ${ }^{1}$ Federal University of ABC, CCNH, Rua Santa Adélia 166, \\ CEP 09210-170, Santo André, Brazil. \\ ${ }^{2}$ Department of Theoretical Physics and IFIC, \\ University of Valencia-CSIC, E-46100, Valencia, Spain.
}

\begin{abstract}
Dynamical gluon mass generation has been traditionally plagued with seagull divergences, and all regularization procedures proposed over the years yield finite but scheme-dependent gluon masses. In this work we show how such divergences can be eliminated completely by virtue of a characteristic identity, valid in dimensional regularization. The ability to trigger the aforementioned identity hinges crucially on the particular Ansatz employed for the three-gluon vertex entering into the Schwinger-Dyson equation governing the gluon propagator. The use of the appropriate threegluon vertex brings about an additional advantage: one obtains two separate (but coupled) integral equations, one for the effective charge and one for the gluon mass. This system of integral equations has a unique solution, which unambiguously determines these two quantities. Most notably, the effective charge freezes in the infrared, and the gluon mass displays power-law running in the ultraviolet, in agreement with earlier considerations.

PACS numbers: 12.38.Lg, 12.38.Aw 12.38.Gc
\end{abstract}




\section{INTRODUCTION}

The dynamical generation of a non-perturbative gluon mass has been first proposed by Cornwall [1], and has received significant attention over the years, both from the theoretical and the phenomenological point of view (see, e.g., [2]). According to this picture, even though the gluon is massless at the level of the fundamental Lagrangian, and remains massless to all order in perturbation theory, the non-perturbative QCD dynamics generate an effective, momentum-dependent mass, without affecting the local $S U(3)_{c}$ invariance, which remains intact. The generation of such a mass has been established by studying the SchwingerDyson equations (SDEs) [3, 4] of QCD, in a gauge-invariant framework based on the pinch technique (PT) [1, 5, 6, 7, 8], and its profound correspondence with the background field method (BFM) [9].

Specifically, when studying the SDE for the PT-BFM gluon propagator, $\Delta\left(q^{2}\right)$, one looks for infrared finite solutions, i.e. with $\Delta^{-1}(0)>0$ (see, e.g., [1, 10, 11, 12]). Such solutions may be fitted by "massive" propagators of the form $\Delta^{-1}\left(q^{2}\right)=q^{2}+m^{2}\left(q^{2}\right)$, where $m^{2}\left(q^{2}\right)$ is not "hard", but depends non-trivially on the momentum transfer $q^{2}$. In order to obtain massive solutions gauge-invariantly, it is necessary to invoke the well-known Schwinger mechanism [13, 14]. In particular, one assumes that the strong QCD dynamics give rise to longitudinally-coupled composite (bound-state) massless poles [15, 16, 17, 18, 19, 20]. These poles play a role rather like Goldstone excitations, in the sense that they preserve the form of the Ward identities satisfied by the Green's functions of the theory in the presence of a mass, but they are not associated with the breaking of any local or global symmetry.

When the renormalization-group logarithms are properly taken into account in the SDE analysis, one obtains, in addition, the non-perturbative generalization of $\alpha\left(q^{2}\right)$, the QCD running coupling (effective charge), of the form [1, 21, 22] $\alpha^{-1}\left(q^{2}\right)=b \ln \left(\frac{q^{2}+4 m^{2}\left(q^{2}\right)}{\Lambda^{2}}\right)$. The presence of $m^{2}\left(q^{2}\right)$ in the argument of the logarithm tames the Landau singularity associated with the perturbative $\beta$ function, and the resulting effective charge is asymptotically free in the ultraviolet, "freezing" at a finite value in the infrared, namely $\alpha^{-1}(0)=b \ln \left(4 m^{2}(0) / \Lambda^{2}\right)$.

As has been emphasized in the literature [23, 24], the generation of a gluon mass is intimately connected with a variety of other related phenomena, and most importantly with the center vortex picture of confinement [25, 26, 27]. In particular, an effective low-energy field theory for describing the gluon mass is the gauged non-linear sigma model known as 
"massive gauge-invariant Yang-Mills" [23]. This model admits vortex solutions, with a longrange pure gauge term in their potentials, which endows them with a topological quantum number corresponding to the center of the gauge group $\left[Z_{N}\right.$ for $\left.S U(N)\right]$, and is, in turn, responsible for quark confinement and gluon screening [24] Specifically, center vortices of thickness $\sim m^{-1}$ are assumed to form a condensate because their entropy (per unit size) is larger than their action. This condensation furnishes an area law to the fundamental representation Wilson loop, thus confining quarks [28].

The general picture described above appears to be in qualitative agreement with a plethora of lattice simulations, where the gluon propagators (in various gauges) reach a finite (non-vanishing) value in the deep infrared, as would happen in the presence of a "mass" [29]. This rather characteristic behavior was already observed in early studies [30], and has been firmly established recently (in the Landau gauge) using large-volume lattices, for both $S U(2)$ [31] and $S U(3)$ [32, 33, 34] pure Yang-Mills (no quarks included).

It is also important to mention that a qualitatively similar situation emerges within the "refined" Gribov-Zwanziger formalism, presented in [35]. In this latter framework the gluon mass is obtained through the addition of appropriate condensates to the original Gribov-Zwanziger action [36, 37]. Interestingly enough, one obtains a gluon mass displaying power-law running, in agreement with a variety of independent studies [11, 38]), as well as the results of the present article, as explained below.

Since the dynamical generation of an effective gluon mass is a purely non-perturbative effect, its technical implementation is rather intricate, and requires the harmonious synthesis of several ingredients [15, 16, 17, 18, 19, 20]. In particular, the exact way how the Schwinger's mechanism will be employed is crucial for the self-consistency of the entire picture. Turns out that one characteristic drawback in the realization of this dynamical scenario can in fact be traced back to a certain subtlety in the implementation of the Schwinger's mechanism at the level of the relevant SDE.

Specifically, the massless poles necessary for triggering Schwinger's mechanism and allowing the possibility of a non-vanishing $\Delta^{-1}(0)$, enter into the SDE for the gluon propagator through the particular Ansatz employed for the fully-dressed three-gluon vertex. Of course, a physically motivated Ansatz must satisfy, in addition, the correct WI, in order to preserve the transversality of the gluon self-energy. Even though several such Ansätze have been proposed over the years [1, 10, 11, 12], they all suffer from a typical problem: as desired, 
$\Delta^{-1}(0)$ does not vanish; however, its value is expressed in terms of seagull integrals, i.e. divergent integrals of the type $\int_{k} \Delta(k)$ and $\int_{k} k^{2} \Delta^{2}(k)$. This fact, in turn, introduces the need to make sense out of these divergences, given that one is not allowed to absorb them into a counterterm of the type $m_{0}^{2}\left(\Lambda_{\mathrm{UV}}^{2}\right) A_{\mu}^{2}$, because this would compromise the gauge invariance of the original Lagrangian, which at no point is to be modified. Even though a variety of regularizations have been proposed in the literature cited above, it is clear that the appearance of these divergences, the need to regularize them, and the ambiguities resulting in from such a regularization, are without a doubt some of the weakest theoretical points of this entire construction.

In this paper we present a more refined Ansatz for the three-gluon vertex, which completely eliminates all seagull divergences. This new Ansatz is inspired from the photon-scalar vertex of scalar QED, introduced by Ball and Chiu [39]. When inserted into the gluon SDE obtained within the PT-BFM formalism, leads to the elimination of all seagull divergences, by triggering a special identity, valid in dimensional regularization (DR), yielding finally a non-vanishing and finite value for $\Delta^{-1}(0)$.

In the context of scalar QED, the identity in question, given in Eq. (3.14), is instrumental in enforcing the masslessness of the photon, in the absence of any bound-state poles, i.e. when the Schwinger mechanism is not in operation. Specifically, the aforementioned Ansatz of [39], when incorporated into the SDE for the photon, gives rise to a $\Delta^{-1}(0)$ that is expressed in terms of seagull contributions, which do not vanish individually, due to the simple fact that the charged scalars are massive already at tree level. However, the vertex of Ball and Chiu is such that the divergent seagull terms appear precisely in the unique combination that will lead to their mutual annihilation, due to the identity of Eq. (3.14).

The proposed three-gluon vertex consists of two parts: (i) a part that leads to the cancellation of all seagull divergences by virtue of the identity of Eq. (3.14), exactly as happens in the scalar QED case; the only difference is that now the seagull terms in question originate from the gluonic self-interactions, i.e. they are composed by the (effectively massive) gluon propagator. (ii) a part that contains massless bound-state poles, thus enforcing the Schwinger mechanism. It is from this second part of the vertex that, after solving the resulting integral equation, one finally obtains a finite value for $\Delta^{-1}(0)$.

In addition to eliminating the seagull divergences, the use of the aforementioned vertex brings about a further important advantage. Specifically, the SDE for the gluon propaga- 
tor of the PT-BFM may be separated unambiguously into two distinct but coupled integral equations, one governing the evolution of the effective charge (running coupling), $\bar{g}^{2}\left(q^{2}\right)$, and one determining the momentum-dependence of the effective gluon mass, $m^{2}\left(q^{2}\right)$. This is to be contrasted with the standard procedure followed in the literature, where the SDE equation is solved for the renormalization-group (RG) invariant combination $\widehat{d}\left(q^{2}\right)=g^{2} \Delta\left(q^{2}\right)$, which is subsequently decomposed into an effective charge and a running mass according to $\widehat{d}\left(q^{2}\right)=\bar{g}^{2}\left(q^{2}\right) /\left(q^{2}+m^{2}\left(q^{2}\right)\right)$, by imposing physically motivated constraints on the form of $\bar{g}^{2}\left(q^{2}\right)$ and $m^{2}\left(q^{2}\right)$. This procedure suffers from the obvious ambiguity of trying to extract two components out of a given function; instead, the new procedure, involving two individual equations, furnishes uniquely $\bar{g}^{2}\left(q^{2}\right)$ and $m^{2}\left(q^{2}\right)$, and it is the $\widehat{d}\left(q^{2}\right)$ that is subsequently obtained uniquely, by combining these two quantities.

The present article is organized as follows. In Section II we review the salient features of dynamical gauge-boson mass generation through the Schwinger mechanism, which constitutes the cornerstone of the entire approach. We explain how the aforementioned mechanism must be judiciously incorporated into the SDE equations of QCD, and the crucial role played by the three-gluon vertex. The problem of the seagull divergences, which is endemic to all existing approaches, is discussed, and some examples of (not fully satisfactory) attempts for its resolution are mentioned. In Section III we turn to the instructive case of scalar QED, and demonstrate in detail how the seagull divergences cancel out from the SDE for the photon propagator, by virtue of the identity of Eq. (3.14), which is in turn triggered by the vertex Ansatz of [39]. A counter-example of a vertex that does not trigger the identity is also discussed. In Section [V] we apply the lessons of the previous section to the case of (quarkless) QCD. In particular, an improved Ansatz for the three-gluon vertex is constructed, which incorporates the Schwinger mechanism through the appearance of massless poles, and, at the same time, triggers the identity of Eq. (3.14), leading to total seagull annihilation. In Section $\nabla$ we obtain the system of two coupled integral equations that determine the momentum dependence of two RG-invariant quantities, namely $\bar{g}^{2}\left(q^{2}\right)$ and $m^{2}\left(q^{2}\right)$, for the entire range of physical momenta, i.e. from the deep IR to the deep UV. The system is solved numerically and the obtained solutions are discussed. Most notably, $m^{2}\left(q^{2}\right)$ display power-law running, in agreement with various earlier considerations. Finally, in Section VI we summarize our conclusions. In addition, in three Appendices we derive in detail various intermediate results used throughout the article. 


\section{MASS GENERATION AND THE PROBLEM OF SEAGULL DIVERGENCES.}

The gluon propagator, $\Delta_{\mu \nu}(q)$, in covariant gauges (in particular, linear, $R_{\xi}$-type of gauges, and the BFM) has the form

$$
\Delta_{\mu \nu}(q)=-i\left[\mathrm{P}_{\mu \nu}(q) \Delta\left(q^{2}\right)+\xi \frac{q_{\mu} q_{\nu}}{q^{4}}\right],
$$

where $\xi$ denotes the gauge-fixing parameter, and the transverse projector $\mathrm{P}_{\mu \nu}(q)$ is given by

$$
\mathrm{P}_{\mu \nu}(q)=g_{\mu \nu}-\frac{q_{\mu} q_{\nu}}{q^{2}}
$$

The scalar factor $\Delta\left(q^{2}\right)$ is given by

$$
\Delta^{-1}\left(q^{2}\right)=q^{2}+i \Pi\left(q^{2}\right)
$$

where $\Pi_{\mu \nu}(q)=\mathrm{P}_{\mu \nu}(q) \Pi\left(q^{2}\right)$ is the gluon self-energy. One usually defines the dimensionless vacuum polarization, to be denoted by $\Pi\left(q^{2}\right)$, as $\Pi\left(q^{2}\right)=q^{2} \Pi\left(q^{2}\right)$, and thus

$$
\Delta^{-1}\left(q^{2}\right)=q^{2}\left[1+\Pi\left(q^{2}\right)\right]
$$

As Schwinger pointed out long time ago [13], the gauge invariance of a vector field does not necessarily imply zero mass for the associated particle, if the current vector coupling is sufficiently strong. Schwinger's fundamental observation was that if (for some reason) the vacuum polarization of the gauge bosons acquires a pole at zero momentum transfer, then the vector meson becomes massive, even if the gauge symmetry forbids a mass at the level of the fundamental Lagrangian [14]. Indeed, casting the self-energy in the form of (2.4), it is clear that if $\Pi\left(q^{2}\right)$ has a pole at $q^{2}=0$ with positive residue $\mu^{2}$, i.e. $\Pi\left(q^{2}\right)=\mu^{2} / q^{2}$, then (in Euclidean space)

$$
\Delta^{-1}\left(q^{2}\right)=q^{2}+\mu^{2}
$$

Thus, the vector meson becomes massive, $\Delta^{-1}(0)=\mu^{2}$, even though it is massless in the absence of interactions $(g=0)$.

There is no physical principle which would preclude $\Pi\left(q^{2}\right)$ from acquiring a pole [40]. Actually, the appearance of the required pole may happen for purely dynamical reasons, and, in particular, without the need to introduce fundamental scalar field in the Lagrangian [41]. Since bound states are expected to exist in most physical systems one may suppose that, for sufficiently strong binding, the mass of such a bound state will be reduced to zero, thus 
generating a mass for the vector meson without interfering with gauge invariance [15, 16, 17, 18, 19, 20].

When applying the dynamical concepts described above to pure Yang-Mills theories, such as quarkless QCD, one assumes that, in a strongly-coupled gauge theory longitudinally coupled zero-mass bound-state excitations are dynamically produced [42]. Thus, it is clear that a vital ingredient for this scenario is strong coupling, which can only come from the infrared instabilities of a non-abelian gauge theories. The aforementioned excitations are like dynamical Nambu-Goldstone bosons, in the sense that they are massless, composite, and longitudinally coupled; but, at the same time, they differ from Nambu-Goldstone bosons as far as their origin is concerned: they do not originate from the spontaneous breaking of any global symmetry. The main role of these excitations is to trigger the Schwinger mechanism, i.e. to provide the required pole in the gluon self-energy, and more specifically, the gaugeindependent $\boldsymbol{\Pi}\left(q^{2}\right)$ obtained with the PT, thus furnishing a gauge-independent dynamical mass for the gluons [43].

Of course, in order to obtain the full dynamics, such as, for example, the momentumdependence of the dynamical mass, one must turn eventually to the SDE that governs the corresponding gauge-boson self-energy. The way the Schwinger mechanism is integrated into the SDE is through the form of the three-gluon vertex. The latter, even in the absence of mass generation, constitutes a central ingredient of the SDE, and plays a crucial role in enforcing the transversality of the gluon self-energy. Therefore, an important requirement for any self-consistent Ansatz used for that vertex is that it should satisfy the correct WI (or STI) of the PT-BFM formulation, namely

$$
q^{\mu} \widetilde{\Gamma}_{\mu \alpha \beta}=\Delta_{\alpha \beta}^{-1}(k+q)-\Delta_{\alpha \beta}^{-1}(k) .
$$

In addition, in order to generate a dynamical mass one must assume that the vertex contains dynamical poles, which will trigger the Schwinger mechanism when inserted into the SDE for the gluon self-energy.

The point is that the full realization of the procedure outlined above is very subtle. In particular, even though the use of a three-gluon vertex containing massless poles and satisfying the correct WI leads indeed to a transverse and infrared finite self-energy (i.e. $\left.\Delta^{-1}(0) \neq 0\right)$, as expected, the actual value of $\Delta^{-1}(0)$ has always been expressed in terms of 
divergent integrals, of the form (see, e.g., [1, 10, 11, 12])

$$
\Delta^{-1}(0)=c_{1} \int_{k} \Delta(k)+c_{2} \int_{k} k^{2} \Delta^{2}(k),
$$

where (in DR) $\int_{k} \equiv \mu^{2 \varepsilon}(2 \pi)^{-d} \int d^{d} k$, with $d=4-\epsilon$ the dimension of space-time [44]. This is not a problem, in principle, provided that the divergent integrals appearing on the rhs of (2.7) can be properly regulated and made finite, without introducing counterterms of the form $m_{0}^{2}\left(\Lambda_{\mathrm{UV}}^{2}\right) A_{\mu}^{2}$, which are forbidden by the local gauge invariance of the fundamental QCD Lagrangian. However, various regularization procedures introduced in the literature have been eventually thwarted by all sorts of additional complications of variable severity.

The simplest regularization possibility, for example, is to employ the usual DR trick for eliminating quadratic divergences, namely subtract $\int_{k} k^{-2}=0$. Assuming a form $\Delta(k)=k^{2}+m^{2}(k)$, this standard (and completely legitimate) operation,

$$
\int_{k} \Delta(k)=\int_{k} \frac{1}{k^{2}+m^{2}(k)}-\int_{k} \frac{1}{k^{2}}=-\int_{k} \frac{m^{2}(k)}{k^{2}\left[k^{2}+m^{2}(k)\right]},
$$

leads to a finite integral, provided $m^{2}(k)$ drops off sufficiently fast in the UV, a feature which is in any case expected from a dynamically generated mass. The general problem with this procedure, however, is the reversal of sign that it induces [1], which eventually clashes with the requirement of a positive-definite $\Delta^{-1}(0)$.

In a recent work [12] the aforementioned procedure was refined in such a way as to evade the sign problem. The general idea is to eliminate the perturbative tail of $\Delta(k)$ by subtracting out DR "zeros", using the generalized formula

$$
\int_{k} \frac{\ln ^{n} k^{2}}{k^{2}}=0, \quad n=0,1,2, \ldots
$$

Specifically, for large enough $k^{2}, \Delta\left(k^{2}\right)$ goes over to its perturbative expression, to be denoted by $\Delta_{\text {pert }}\left(k^{2}\right)$; it has the form

$$
\Delta_{\text {pert }}\left(k^{2}\right)=\sum_{n=0}^{N} a_{n} \frac{\ln ^{n} k^{2}}{k^{2}},
$$

where the coefficient $a_{n}$ are known from the perturbative expansion. Then one may use (2.9) to regularize the rhs of (2.7), and obtain

$$
16 \pi^{2} \Delta_{\text {reg }}^{-1}(0)=c_{1} \int_{0}^{s} d y y\left[\Delta(y)-\Delta_{\text {pert }}(y)\right]+c_{2} \int_{0}^{s} d y y^{2}\left[\Delta^{2}(y)-\Delta_{\text {pert }}^{2}(y)\right],
$$

which is finite (and has been shown to be positive). As explained in [12], the obvious ambiguity of this procedure is the choice of the point $s$, past which the two curves, $\Delta(y)$ 

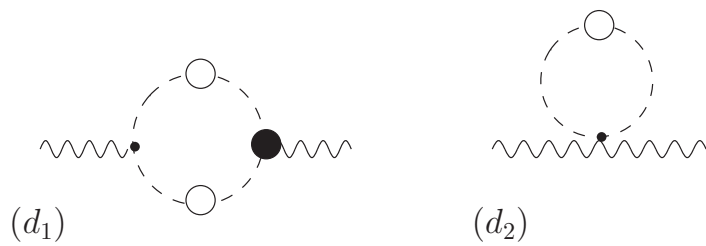

$\left(d_{2}\right)$
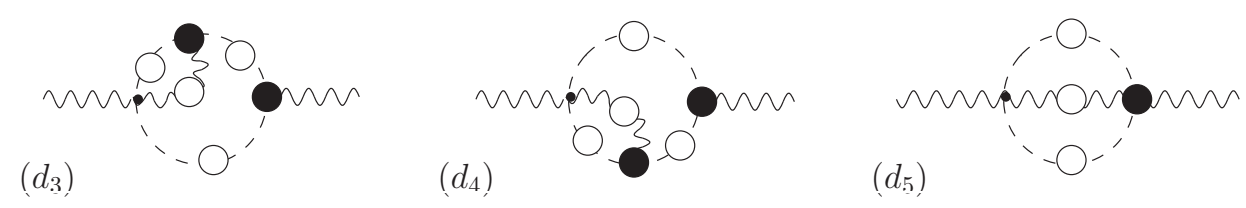

FIG. 1: Diagrams contributing to the SDE for the photon self-energy in scalar QED.

and $\Delta_{\text {pert }}(y)$, are assumed to coincide (and cancel exactly against each other). Thus, the actual value of $\Delta_{\text {reg }}^{-1}(0)$ remains largely undetermined. Even though additional qualitative arguments may be used to restrict the allowed interval of $s$, thus reaching good agreement with recent lattice data, from the theoretical point of view it is clear that this issue is far from settled.

\section{SCALAR QED AND THE SEAGULL IDENTITY}

In this section we will study some of the basic issues related to the appearance and cancellation of seagull divergences in the context of a theory much simpler than QCD, namely scalar QED. Specifically, we will study the SDE governing the photon, and we will discover a basic identity, which, in the absence of massless poles (i.e., with the Schwinger mechanism "switched off") enforces the masslessness of the photon, despite the fact that individual seagull contributions do not vanish. In addition, we will see through an explicit detailed construction that the Ansätze employed for the all-order photon-scalar vertex entering into the SDE are crucial for the activation of this identity.

The SDE for the photon of scalar QED is shown in Fig. 1. It is a straightforward exercise to demonstrate that, by virtue of the Abelian WI's satisfied by the full vertices of the theory, the SDE may be truncated "loop-wise", without compromising the transversality of the photon, i.e., $q^{\mu} \Pi_{\mu \nu}^{\left[\left(d_{1}\right)+\left(d_{2}\right)\right]}=q^{\mu} \Pi_{\mu \nu}^{\left[\left(d_{3}\right)+\left(d_{4}\right)+\left(d_{5}\right)\right]}=0$.

At the "one-loop dressed" level the SDE for the photon self-energy reads (Fig. 21)

$$
\Pi_{\mu \nu}(q)=e^{2} \int_{k} \Gamma_{\mu}^{(0)} \mathcal{D}(k) \mathcal{D}(k+q) \Gamma_{\nu}+e^{2} \int_{k} \Gamma_{\mu \nu}^{(0)} \mathcal{D}(k),
$$



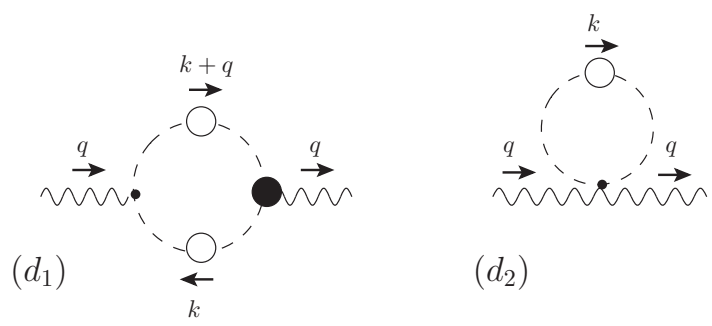

$\left(d_{2}\right)$

FIG. 2: The "one-loop dressed" SDE for the photon self-energy.

where $\mathcal{D}(k)$ is the fully-dressed propagator of the scalar field. $\Gamma_{\nu}$ is the fully dressed photonscalar vertex, whose tree-level expression is given by $\Gamma_{\mu}^{(0)}=-i(2 k+q)_{\mu}$. Moreover, the bare quadrilinear photon-scalar vertex is given by $\Gamma_{\mu \nu}^{(0)}=2 i g_{\mu \nu}$. The photon-scalar vertex $\Gamma_{\mu}$ and the scalar propagator $\mathcal{D}$ are related by the Abelian all-order WI

$$
q^{\nu} \Gamma_{\nu}=\mathcal{D}^{-1}(k+q)-\mathcal{D}^{-1}(k)
$$

It is fairly easy to demonstrate the transversality of $\Pi_{\mu \nu}(q)$, namely that $q^{\nu} \Pi_{\mu \nu}(q)=0$. To that end, act with $q^{\nu}$ on the two terms on the rhs of (3.1), and notice that, by virtue of (3.2) , the (contracted) first term (after appropriate shifting of the integration variable, a legitimate operation in DR), cancels exactly against the second. Given that $\Pi_{\mu \nu}(q)$ is transverse, it assumes the form $\Pi_{\mu \nu}(q)=\Pi\left(q^{2}\right) \mathrm{P}_{\mu \nu}(q)$; thus one may determine $\Pi\left(q^{2}\right)$ simply by taking the trace of both sides of (3.1), i.e.

$$
\Pi\left(q^{2}\right)=\frac{-2 i e^{2}}{d-1}\left[\int_{k} \mathcal{D}(k) \mathcal{D}(k+q) k^{\mu} \Gamma_{\mu}-d \int_{k} \mathcal{D}(k)\right],
$$

where Eq. (3.2) was used.

Let us compute from (3.3) the one-loop expression for $\Pi\left(q^{2}\right)$, to be denoted by $\Pi^{(1)}\left(q^{2}\right)$. We have (we are using DR throughout)

$$
\Pi^{(1)}\left(q^{2}\right)=\frac{-i e^{2}}{d-1}\left[\int_{k}\left(4 k^{2}-q^{2}\right) \mathcal{D}_{0}(k) \mathcal{D}_{0}(k+q)-2 d \int_{k} \mathcal{D}_{0}(k)\right],
$$

where $\mathcal{D}_{0}(k)=\left(k^{2}-m^{2}\right)^{-1}$. Taking the limit $q \rightarrow 0$, we find

$$
\Pi^{(1)}(0)=\frac{-4 i e^{2}}{d-1}\left[\int_{k} k^{2} \mathcal{D}_{0}^{2}(k)-\frac{d}{2} \int_{k} \mathcal{D}_{0}(k)\right] .
$$

Of course, there is no doubt that the photon remains massless perturbatively, i.e. we must have that $\Pi^{(1)}(0)=0$. However, the way this requirement is realized is rather subtle: the rhs of (3.5) vanishes indeed, by virtue of an identity that is exact in DR, namely

$$
\int_{k} \frac{k^{2}}{\left(k^{2}-m^{2}\right)^{2}}=\frac{d}{2} \int_{k} \frac{1}{k^{2}-m^{2}}
$$


or, equivalently,

$$
2 m^{2} \int_{k} \frac{1}{\left(k^{2}-m^{2}\right)^{2}}=(d-2) \int_{k} \frac{1}{k^{2}-m^{2}} .
$$

The relations given in (3.6) and (3.7) can be easily verified using the standard integration rules of the DR [45]. Thus, the perturbative masslessness of the photon is explicitly realized and self-consistently enforced within the DR. Note that Eq.(3.6) may be cast in a form that is particularly suggestive for the analysis that follows, namely

$$
\int_{k} k^{2} \frac{\partial \mathcal{D}_{0}(k)}{\partial k^{2}}=-\frac{d}{2} \int_{k} \mathcal{D}_{0}(k)
$$

To demonstrate (3.8) directly, i.e. without deducing it from (3.6), we first go to Euclidean space, use spherical coordinates (see A2), and integrate by parts (in $d$ dimensions). Setting $k_{E}^{2}=y$, we have (suppressing the angular integral)

$$
\int_{0}^{\infty} d y y^{\frac{d}{2}} \frac{\partial \mathcal{D}_{0}(y)}{\partial y}=\left[y^{\frac{d}{2}} \mathcal{D}_{0}(y)\right]_{0}^{\infty}-\frac{d}{2} \int_{0}^{\infty} d y y^{\left(\frac{d}{2}-1\right)} \mathcal{D}_{0}(y)
$$

Evidently, dropping the surface term, an operation that can be formally justified by the standard analytic continuation employed within the DR (see, e.g. [46]), yields immediately Eq. (3.8).

We now return to the general Eq.(3.3). In order to analyze it further we must furnish some information about the form of $\Gamma_{\mu}$. Of course, any meaningful Ansatz for $\Gamma_{\mu}$ must satisfy the WI of (3.2), or else the transversality of $\Pi_{\mu \nu}(q)$ will be compromised from the outset. The form obtained by Ball and Chiu [39], after "solving" the WI, under the additional requirement of not introducing kinematic singularities, is

$$
\Gamma_{\mu}=\frac{(2 k+q)_{\mu}}{(k+q)^{2}-k^{2}}\left[\mathcal{D}^{-1}(k+q)-\mathcal{D}^{-1}(k)\right]+A(k, q)\left[(k+q) \cdot q k_{\mu}-k \cdot q(k+q)_{\mu}\right]
$$

where $A(k, q)$ is finite as $q \rightarrow 0$. Clearly the first term satisfies (3.2), while the part proportional to $A(k, q)$ is identically conserved.

It is easy to recognize that when this latter term is inserted into (3.3) it yields a contribution that vanishes as $q \rightarrow 0$, with no additional assumptions, other than the regular nature of $A(k, q)$; we will therefore neglect that term in what follows. On the other hand, the first term of $\Gamma_{\mu}$ yields

$$
\Pi\left(q^{2}\right)=\frac{i e^{2}}{d-1}\left[\int_{k}\left(4 k^{2}-q^{2}\right) \frac{\mathcal{D}(k+q)-\mathcal{D}(k)}{(k+q)^{2}-k^{2}}+2 d \int_{k} \mathcal{D}(k)\right],
$$


Taking the limit of Eq.(3.11) as $q \rightarrow 0$, using that

$$
\frac{\mathcal{D}(k+q)-\mathcal{D}(k)}{(k+q)^{2}-k^{2}} \rightarrow \frac{\partial \mathcal{D}(k)}{\partial k^{2}}+\mathcal{O}\left(q^{2}\right)
$$

we have that

$$
\Pi(0)=\frac{4 i e^{2}}{d-1}\left[\int_{k} k^{2} \frac{\partial \mathcal{D}(k)}{\partial k^{2}}+\frac{d}{2} \int_{k} \mathcal{D}(k)\right],
$$

Of course, we must have that $\Pi(0)=0$, given that there is nothing in the dynamics that could possibly endow the photon with a mass; in particular, we have not employed Schwinger's mechanism, i.e. we have not introduced dynamical poles, and, given the form of (3.10), neither kinematic ones, which might simulate the dynamical ones at the level of the SDE (see below). Thus, the rhs of (3.13) must vanish, and therefore, we must have that

$$
\int_{k} k^{2} \frac{\partial \mathcal{D}(k)}{\partial k^{2}}=-\frac{d}{2} \int_{k} \mathcal{D}(k)
$$

which is the non-perturbative generalization of (3.8); its demonstration proceeds exactly in the same way (and under the same assumptions).

Note a crucial point: the seagull terms appearing in (3.8) cannot be set to zero individually, because the scalar propagator inside them is massive (already at tree-level): the only way to keep the photon massless, is to employ (3.8), which cancels them against each other. For example, if the term $\int_{k} \mathcal{D}(k)$ on the rhs were multiplied by any factor other than $(d / 2)$ one would be stuck with seagull divergences.

Let us now try a different Ansatz for $\Gamma_{\mu}$, which, due to its special form will not trigger Eq. (3.14), and thus will lead to a non-vanishing (but divergent) value for $\Pi(0)$. Specifically, consider the vertex given by

$$
\Gamma_{\mu}=\Gamma_{\mu}^{(0)}+\frac{q_{\mu}}{q^{2}}[\Sigma(k+q)-\Sigma(k)]
$$

where $\Sigma(k)$ is the self-energy of the scalar field, $\mathcal{D}^{-1}(k)=k^{2}-m^{2}+\Sigma(k)$. Equivalently, we may write

$$
\Gamma_{\mu}=\left\{\Gamma_{\mu}^{(0)}-\frac{q_{\mu}}{q^{2}}\left[(k+q)^{2}-k^{2}\right]\right\}+\frac{q_{\mu}}{q^{2}}\left[\mathcal{D}^{-1}(k+q)-\mathcal{D}^{-1}(k)\right] .
$$

The $\Gamma_{\mu}$ in (3.15)-(3.16) satisfies again the WI of (3.2), and thus, as before, the transversality of the vacuum polarization is guaranteed. There is an important difference, however, between (3.10) and (3.16): the latter contains massless poles, and thus, is capable of giving rise to a non-vanishing $\Delta^{-1}(0)$. 
Indeed, substituting $\Gamma_{\mu}$ of Eq. (3.16) into (3.3), after straightforward algebra we obtain

$$
\Pi\left(q^{2}\right)=\frac{4 i e^{2}}{d-1}\left[\int_{k} \mathcal{D}(k) \mathcal{D}(k)(k+q)\left[\frac{(k \cdot q)^{2}}{q^{2}}-k^{2}\right]+\frac{d-1}{2} \int_{k} \mathcal{D}(k)\right],
$$

Using that

$$
\left.\int_{k} \frac{(k \cdot q)^{2}}{q^{2}} \mathcal{D}(k) \mathcal{D}(k+q)\right|_{q^{2} \rightarrow 0}=\frac{1}{4} \int_{k} k^{2} \mathcal{D}^{2}(k),
$$

we find from (3.17) (setting $d=4$ )

$$
\Pi(0)=i e^{2}\left[2 \int_{k} \mathcal{D}(k)-\int_{k} k^{2} \mathcal{D}^{2}(k)\right],
$$

which has the general form given in (2.7). Evidently, the Ansatz of (3.15) does not trigger Eq. (3.14), and one ends up with a non-zero $\Delta^{-1}(0)$, which, however, is expressed in terms of divergent seagull-type integrals.

It is evident from the above analysis that the massless poles, indispensable as they may be for generating a non-vanishing $\Delta^{-1}(0)$, must be incorporated into the SDE with particular care, or else they give rise to seagull divergences. But even without this pathology, it is clear that the vertex of (3.15) does not constitute an optimal Ansatz. For example, if the $\left(1 / q^{2}\right)$ pole is considered to be of purely non-perturbative origin (as it is supposed to), it has vanishing perturbative expansion, and so, to all orders in perturbation theory $\Gamma_{\mu}=\Gamma_{\mu}^{(0)}$, which is of course not correct.

In the next section we will see that the correct procedure is to add to the vertex of (3.10) non-perturbative pole terms, in such a way as to preserve the seagull cancellation implemented by Eq. (3.14), and, at the same time, obtain a finite $\Delta^{-1}(0)$.

\section{FINITE GLUON MASS GENERATION}

After having fixed the ideas in the context of a simple Abelian model, we now turn to a pure Yang-Mills theory. In particular, we will study the SDE of the gluon propagator in the case of pure (quarkless) QCD, within the PT-BFM framework. As has been explained in detail in the recent literature [10, 47, 48], this latter formalism allows for a gauge-invariant truncation of the SD series, in the sense that it preserves manifestly and at every step the transversality of the gluon self-energy. Specifically, for the case at hand, we will consider only the "one-loop dressed" part of the gluon SDE that contains gluons shown in Fig. 3, 

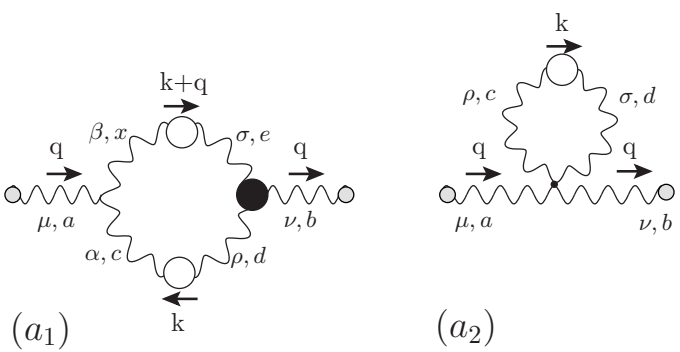

FIG. 3: The "one-loop dressed" gluonic graphs contributing to the SDE for the (background) gluon self-energy, $\widehat{\Pi}_{\mu \nu}(q)$.

leaving out (gauge-invariantly!) the "one-loop dressed" ghost contributions and all "twoloop dressed" diagrams. Note that the Feynman rules used to build the SD series for the (background) gluon self-energy, $\widehat{\Pi}_{\mu \nu}(q)$, are those of the BFM [9]; in particular, the external gluons (distinguished by the grey circles attached to them) are treated as if they were background gluons. The two tree-level vertices necessary for our analysis are given in Fig. 4, as we will see in a moment, the form of these vertices is crucial for obtaining from the SDE precisely the right combination of terms (and with the correct relative weights) that appears in (3.14).

In order to reduce the algebraic complexity of the problem, we drop the longitudinal terms from the gluon propagators inside the integrals, i.e. we set $\Delta_{\alpha \beta} \rightarrow-i g_{\alpha \beta} \Delta$ [49]. This does not compromise the transversality of $\widehat{\Pi}_{\mu \nu}(q)$ provided that we do the same on the rhs

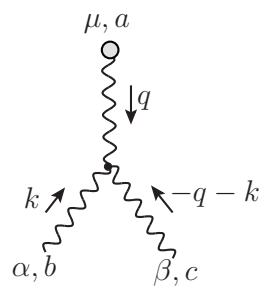

$$
\begin{aligned}
g f^{a b c}\left[(2 k+q)_{\mu} g_{\alpha \beta}+2 q_{\beta} g_{\mu \alpha}-2 q_{\alpha} g_{\mu \beta}\right], \\
-i g^{2} \quad\left[f^{a b x} f^{x c d}\left(g_{\mu \alpha} g_{\nu \beta}-g_{\mu \beta} g_{\nu \alpha}+g_{\mu \nu} g_{\alpha \beta}\right)\right. \\
\quad+f^{a d x} f^{x b c}\left(g_{\mu \nu} g_{\alpha \beta}-g_{\mu \alpha} g_{\nu \beta}+g_{\mu \beta} g_{\nu \alpha}\right) \\
\left.\quad+f^{a c x} f^{x b d}\left(g_{\mu \nu} g_{\alpha \beta}-g_{\mu \beta} g_{\nu \alpha}\right)\right] .
\end{aligned}
$$

FIG. 4: The trilinear and quadrilinear gluon vertices in the Feynman gauge of BFM. 
of the WI satisfied by $\widetilde{\Gamma}_{\nu \alpha \beta}$, namely we have simply

$$
q^{\nu} \widetilde{\Gamma}_{\nu \alpha \beta}=\left[\Delta^{-1}(k+q)-\Delta^{-1}(k)\right] g_{\alpha \beta}
$$

instead of the full WI given in (2.6).

Then, it is straightforward to show that the SDE corresponding to Fig. 3 reduces to

$$
\widehat{\Delta}^{-1}(q)=q^{2}+\frac{i g^{2} C_{\mathrm{A}}}{2(d-1)}\left[\int_{k} \widetilde{\Gamma}_{\mu \alpha \beta}^{(0)} \Delta(k) \Delta(k+q) \widetilde{\Gamma}^{\mu \alpha \beta}+2 d^{2} \int_{k} \Delta(k)\right],
$$

where $C_{\mathrm{A}}$ the Casimir eigenvalue of the adjoint representation $\left[C_{\mathrm{A}}=N\right.$ for $\left.S U(N)\right]$. $\widetilde{\Gamma}_{\mu \alpha \beta}^{(0)}(q, k,-k-q)$ is the bare three-gluon vertex in the Feynman gauge of the BFM, given in Fig. 4, and $\widetilde{\Gamma}_{\mu \alpha \beta}$ denotes its fully-dressed version.

The function $\widehat{\Delta}(q)$ appearing on the lhs of (4.4) is the scalar part of the gluon propagator in the BFM, i.e. two background gluons entering; its relation to the self-energy $\widehat{\Pi}_{\mu \nu}(q)$ is the same as in (2.3). Note that $\widehat{\Delta}(q)$ is related to the standard $\Delta(q)$, defined in the $R_{\xi}$ gauges, by means of the powerful identity, namely $\widehat{\Delta}(q)\left[1+G\left(q^{2}\right)\right]^{2}=\Delta(q)$, where $G\left(q^{2}\right)$ is an auxiliary two-point function [6, 50] whose dynamics have been studied in detail in the recent literature (see, e.g. [51], and references therein). To further simplify the problem, without compromising its essential features, we will next set $G\left(q^{2}\right)=0$, i.e. we effectively assume that, inside the quantum loops, $\Delta(q)=\widehat{\Delta}(q)$. Thus, in what follows we will be dealing with a single propagator, namely $\widehat{\Delta}(q)$, and will suppress the "hats" in order to reduce the notation.

\section{A. The three-gluon vertex}

Up until this point the analysis presented in this section is completely standard within the PT-BFM framework. At this point enters a new ingredient, namely the judicious Ansatz for the three-gluon vertex which, in addition to satisfying (4.3) will allow us to use the seagull identity (3.14) and get a non-vanishing and finite $\Delta^{-1}(0)$.

To begin with, let us first write $\Delta^{-1}(q)$ in the alternative form (in Minkowski space)

$$
\Delta^{-1}(q)=q^{2} H^{-1}(q)-\widetilde{m}^{2}(q)
$$

The tree-level result for $\Delta^{-1}(q)$ is recovered by setting $H^{-1}(q)=1$ and $\widetilde{m}^{2}=0$. 


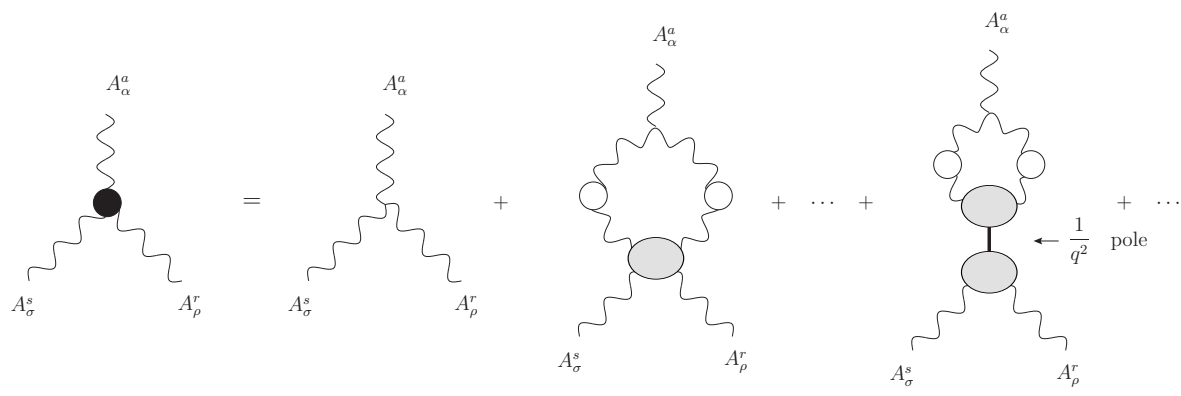

FIG. 5: The SDE for the three-gluon vertex. All kernels are one-particle irreducible, and the $1 / q^{2}$ pole is not kinematic but dynamical (purely non-perturbative); physically it corresponds to a (composite) Goldstone mode, necessary for maintaining the local gauge invariance.

Then, an appropriate Ansatz for $\widetilde{\Gamma}_{\nu \alpha \beta}$ is given by

$$
i \widetilde{\Gamma}_{\mu \alpha \beta}=\left[\frac{(k+q)^{2} H^{-1}(k+q)-k^{2} H^{-1}(k)}{(k+q)^{2}-k^{2}}\right] \widetilde{\Gamma}_{\mu \alpha \beta}^{(0)}+V_{\mu \alpha \beta},
$$

where the term $V_{\mu \alpha \beta}$ contains the non-perturbative contributions due to bound-state poles associated with the Schwinger mechanism. Thus, $V_{\mu \alpha \beta}$ represents the term containing the $1 / q^{2}$ pole on the rhs in Fig. 5. Note that we must have

$$
q^{\mu} V_{\mu \alpha \beta}=\left[\widetilde{m}^{2}(k)-\widetilde{m}^{2}(k+q)\right] g_{\alpha \beta},
$$

in order for the $\widetilde{\Gamma}_{\mu \alpha \beta}$ of Eq. (4.6) to satisfy (by construction) the correct WI of (4.3).

The Ansatz of (4.6) mimics that of Eq. (3.10) to the extent that the first term contains the right structure to produce, when inserted into the first term on the rhs of (4.4), the derivative term appearing on the lhs of (3.14). The rhs of (3.14) is already there: it is the second term on the rhs of (4.4), originating directly from the seagull diagram $\left(a_{2}\right)$.

Notice that the first term on the rhs of (4.6) may be expanded perturbatively, whereas $V_{\mu \alpha \beta}$ vanishes perturbatively to all orders. Qualitatively speaking, $H^{-1}(q)$ will have the form (assume for simplicity a constant $\widetilde{m}^{2}$ )

$$
H^{-1}(q) \sim g^{2} \int_{0}^{1} d x \ln \left[q^{2} x(x-1)+\widetilde{m}^{2}\right]+\mathcal{O}\left(g^{4}\right) ;
$$

perturbatively (to all orders), $\widetilde{m}^{2}=0$, and one recovers the usual one-loop logarithm $g^{2} \ln \left(q^{2}\right)$ (displaying the typical Landau pole in the IR). Thus the role of the term $V_{\mu \alpha \beta}$ is two-fold: (i) it tames the Landau pole inside the dimensionless perturbative logarithm, and (ii) as can be seen directly from Eq. (4.5), it can furnish an IR-finite propagator, $\Delta^{-1}(0)=-\widetilde{m}^{2}(0)$ 
(in Minkowski space), provided, of course, that the equation governing $\widetilde{m}^{2}$ has non-trivial solutions (see next section).

An important point related to the form of $\widetilde{\Gamma}_{\mu \alpha \beta}$ is that the transverse (i.e. identically conserved) component associated with the first term in Eq. (4.6) has been set to zero [it would correspond to the term proportional to $A(k, q)$ in Eq. (3.10)]. As is well known, such an omission, while of little importance in the IR, leads to the mishandling of overlapping divergences in the UV. This, in turn, spoils the multiplicative renormalizability of the resulting SDE, which must be renormalized subtractively [see also discussion following Eq. (5.12)].

An analogous Ansatz for the vertex $V_{\mu \alpha \beta}$ may be deduced following a similar philosophy. For example, a simple Ansatz that captures the two essential characteristics of having a (composite), longitudinally coupled poles, and satisfying the WI of (4.7) is

$$
V_{\mu \alpha \beta}=V_{\mu \alpha \beta}^{\ell}+V_{\mu \alpha \beta}^{\mathrm{t}}
$$

where

$$
V_{\mu \alpha \beta}^{\ell}=\frac{q_{\mu}}{q^{2}}\left[\widetilde{m}^{2}(k)-\widetilde{m}^{2}(k+q)\right] g_{\alpha \beta},
$$

and with the transverse part $V_{\mu \alpha \beta}^{\mathrm{t}}$ satisfying

$$
q^{\mu} V_{\mu \alpha \beta}^{\mathrm{t}}=0
$$

We emphasize that, in principle, the form of $V_{\mu \alpha \beta}^{\mathrm{t}}$ may not be chosen at will, but must ideally be determined from solving the corresponding SDE for the three-gluon vertex, shown schematically in Fig.5. Given that this task lies beyond our present powers, in what follows we will treat $V_{\mu \alpha \beta}^{\mathrm{t}}$ as being essentially undetermined [see discussion before Eq. (4.21)].

We can write the vertex of (4.6) equivalently as

$$
i \widetilde{\Gamma}_{\mu \alpha \beta}=\left[\frac{\Delta^{-1}(k+q)-\Delta^{-1}(k)}{(k+q)^{2}-k^{2}}\right] \widetilde{\Gamma}_{\nu \alpha \beta}^{(0)}+\bar{V}_{\mu \alpha \beta},
$$

with

$$
\bar{V}_{\mu \alpha \beta}=V_{\mu \alpha \beta}+V_{\mu \alpha \beta}^{\mathrm{r}}
$$

where

$$
V_{\mu \alpha \beta}^{\mathrm{r}}=(2 k+q)_{\mu}\left[\frac{\widetilde{m}^{2}(k+q)-\widetilde{m}^{2}(k)}{(k+q)^{2}-k^{2}}\right] g_{\alpha \beta} .
$$

The term $V_{\mu \alpha \beta}^{\mathrm{r}}$ is a residual piece, acting as an additional (non-perturbative) vertex term, originating from forcing the vertex to assume the form of (4.12). As we will see shortly, this 
last way of writing $\widetilde{\Gamma}^{\nu \alpha \beta}$ makes the use of the basic identity of Eq.(3.14) immediate. Thus, after these rearrangements, we have that the final non-perturbative effective vertex $\bar{V}_{\mu \alpha \beta}$ must be transverse,

$$
q^{\mu} \bar{V}_{\mu \alpha \beta}=0
$$

In summary, the vertex Ansatz proposed in (4.6) [and (4.12)] above has three important properties: (i) satisfies identically the WIs of (4.3), a fact that ensures the transversality of the resulting gluon self-energy; (ii) the pole term contained in $V_{\mu \alpha \beta}$ makes it possible to have a non-vanishing $\Delta^{-1}(0)$; (iii) triggers the basic equation (3.14), which, in turn, allows one to dispose of the seagull-type terms. Thus, as we will see in the next subsection, the $\Delta^{-1}(0)$ obtained from the SDE is both non-vanishing and finite.

\section{B. The implications for the SDE}

Let us now study the effect that the three-gluon vertex of (4.6) has on the SDE for $\Delta\left(q^{2}\right)$ given in (4.4). Substituting for the $\widetilde{\Gamma}^{\mu \alpha \beta}$ on the rhs the expression given in (4.12) we obtain after simple algebra

$$
\Delta^{-1}\left(q^{2}\right)=q^{2}-\frac{i g^{2} C_{\mathrm{A}}}{2(d-1)}\left[\Pi(q)+\Pi_{\widetilde{m}}(q)\right]
$$

with

$$
\Pi(q)=(7 d-8) q^{2} \int_{k} \frac{\Delta(k+q)-\Delta(k)}{(k+q)^{2}-k^{2}}+4 d\left[\int_{k} k^{2} \frac{\Delta(k+q)-\Delta(k)}{(k+q)^{2}-k^{2}}+\frac{d}{2} \int_{k} \Delta(k)\right],
$$

and

$$
\begin{aligned}
\Pi_{\widetilde{m}}(q) & =\int_{k} \widetilde{\Gamma}_{\mu \alpha \beta}^{(0)} \Delta(k) \Delta(k+q) \bar{V}^{\mu \alpha \beta} \\
& =\int_{k} \widetilde{\Gamma}_{\mu \alpha \beta}^{(0)} \Delta(k) \Delta(k+q)\left[V^{\ell}+\left\{V^{\mathrm{t}}+V^{\mathrm{r}}\right\}\right]^{\mu \alpha \beta}
\end{aligned}
$$

The term in square brackets on the rhs of (4.17) has exactly the structure needed for employing (3.14). In particular, using the notation introduced in (A1), we can write (4.17) as

$$
\Pi(q)=(7 d-8) q^{2} R_{\Delta}(q)+4 d T_{\Delta}(q)
$$

Note the perfect balance of relative coefficients required for the precise term $T_{\Delta}(q)$ to emerge from the SDE. This becomes possible within the PT-BFM framework thanks to the special vertices shown in Fig. 4, Instead, in the conventional SD formulation (e.g., in the $R_{\xi}$ gauges) 
it would be very difficult to obtain the precise combination of terms needed for implementing (3.14).

Perturbatively, at one loop, $\Pi(q)$ of (4.18) reduces to a simple and rather familiar result [of course, $\Pi_{\widetilde{m}}(q)$ vanishes perturbatively, to all orders]. Specifically, setting $\Delta(k)=1 / k^{2}$ on the rhs of (4.17), it is immediate to recognize that the term in square brackets vanishes, since it becomes proportional to the DR integral $\int_{k} k^{-2}=0$, while the first term becomes

$$
\Pi(q)=-(7 d-8) q^{2} \int_{k} \frac{1}{k^{2}(k+q)^{2}} .
$$

This is the one-loop contribution to the gluon self-energy coming from the graphs containing only gluons. Remember that the ghosts have been omitted without interfering with the transversality of the answer; their omission amounts to having in front of the leading logarithm the coefficient $(7 d-8)$ instead of $(7 d-6)$; as a result (at $d=4)$ the coefficient of the one-loop $\beta$ function is $10 C_{\mathrm{A}} / 48 \pi^{2}$ instead of $11 C_{\mathrm{A}} / 48 \pi^{2}[9,47]$.

Let us now turn to the basic non-perturbative features of (4.16). Since by virtue of (3.14) we have that $T_{\Delta}(0)=0$, it is clear that $\Pi(0)=0$. Thus, the part of the calculation determining $\Pi(q)$ is very similar to that of scalar QED, in the sense that it keeps the gluon (photon) massless. On the other hand, the term $\Pi_{\widetilde{m}}(q)$, not present in the scalar QED study, makes it possible to have $\Delta^{-1}(0) \neq 0$ for the gluons.

To see this explicitly, we focus on the $\Pi_{\widetilde{m}}(q)$ given in (4.18). The integral on the rhs of (4.18) receives two contributions, one from the term containing the vertex $V^{\ell}$ [given in (4.10)] and one from the term containing the sum $\left\{V^{\mathrm{t}}+V^{\mathrm{r}}\right\}$. Let now us assume, for simplicity, that the (undetermined) transverse vertex $V^{\mathrm{t}}$ will be such that, when added to $V^{\mathrm{r}}$ [given in (4.14)], will make the contribution from $\left\{V^{\mathrm{t}}+V^{\mathrm{r}}\right\}$ to become numerically subleading compared to that of $V^{\ell}$. For instance, $V^{\mathrm{t}}$ could be such that the total contribution from $\left\{V^{\mathrm{t}}+V^{\mathrm{r}}\right\}$ were proportional to the terms $I_{2}\left(q^{2}\right)$ and $I_{4}\left(q^{2}\right)$, shown to be subleading in (C6). Then, keeping only $V^{\ell}$ in (4.18), we obtain

$$
\begin{aligned}
\Pi_{\widetilde{m}}(q) & =-\frac{2 d}{q^{2}} \int_{k} k^{2} \Delta(k) \Delta(k+q)\left[\widetilde{m}^{2}(k+q)-\widetilde{m}^{2}(k)\right] \\
& =-\frac{2 d}{q^{2}} \int_{k} \widetilde{m}^{2}(k) \Delta(k) \Delta(k+q)\left[(k+q)^{2}-k^{2}\right]
\end{aligned}
$$

Then, from the Appendix C, Eq. (C3), we have that $\Pi_{\widetilde{m}}(0) \neq 0$, which, in turn, gives rise to $\Delta^{-1}(0) \neq 0$, as announced. 
An important consequence of this analysis is that Eq. (4.16) can be split unambiguously into two parts, one that vanishes as $q^{2} \rightarrow 0$ and one that does not. In fact, using (4.5) on the lhs of (4.16), we can assign the two types of contributions into two separate (but coupled) equations, i.e.

$$
\begin{aligned}
q^{2} H^{-1}(q) & =q^{2}-\frac{i g^{2} C_{\mathrm{A}}}{2(d-1)} \Pi(q), \\
\widetilde{m}^{2}(q) & =\frac{i g^{2} C_{\mathrm{A}}}{2(d-1)} \Pi_{\widetilde{m}}(q) .
\end{aligned}
$$

As we will see in the next section, the first equation will determine the momentum dependence of the effective charge, and the second the running of the gluon mass.

\section{COUPLED EQUATIONS FOR EFFECTIVE CHARGE AND GLUON MASS}

In this section we will study the system of integral equations given in Eqs. (4.22)-(4.23), under certain simplifying assumptions. The first step in our analysis consists in rewriting Eqs. (4.22)-(4.23) in terms of RG-invariant quantities, which will correspond to the effective charge and the physical gluon mass. Then, the two coupled equations will be expressed in terms of these two RG-invariant quantities, and will be further evaluated. We will assume a spectral representation for the gluon propagator [viz. Eq. (B7)], a fact that simplifies enormously the form of the resulting equations. Finally, we will solve the system numerically and study the properties of the obtained solutions.

\section{A. RG-invariant quantities}

It is well-known that, due to the Abelian WIs satisfied by the PT-BFM Green's functions, the propagator $\Delta^{-1}\left(q^{2}\right)$ absorbs all the RG logs, exactly as happens in QED with the photon self-energy. Specifically, let us define the renormalization constants of the gauge-coupling and the effective self-energy as

$$
\begin{aligned}
g\left(\mu^{2}\right) & =Z_{g}^{-1}\left(\mu^{2}\right) g_{0}, \\
\Delta\left(q^{2} ; \mu^{2}\right) & =Z_{A}^{-1 / 2}\left(\mu^{2}\right) \Delta_{0}\left(q^{2}\right),
\end{aligned}
$$


where the "0" subscript indicates bare quantities. Then, since the renormalization constants above satisfy the QED-like relation

$$
Z_{g}=Z_{A}^{-1 / 2}
$$

the product

$$
\widehat{d}_{0}\left(q^{2}\right)=g_{0}^{2} \Delta_{0}\left(q^{2}\right)=g^{2} \Delta\left(q^{2}\right)=\widehat{d}\left(q^{2}\right),
$$

retains the same form before and after renormalization, i.e., it forms a RG-invariant ( $\mu$-independent) quantity [1].

For asymptotically large momenta one may extract from $\widehat{d}\left(q^{2}\right)$ a dimensionless quantity by writing,

$$
\widehat{d}\left(q^{2}\right)=\frac{\bar{g}^{2}\left(q^{2}\right)}{q^{2}},
$$

where $\bar{g}^{2}\left(q^{2}\right)$ is the RG-invariant effective charge of QCD; at one-loop

$$
\bar{g}^{2}\left(q^{2}\right)=\frac{g^{2}}{1+b g^{2} \ln \left(q^{2} / \mu^{2}\right)}=\frac{1}{b \ln \left(q^{2} / \Lambda_{\mathrm{QCD}}^{2}\right)} .
$$

where $\Lambda_{\mathrm{QCD}}$ denotes an RG-invariant mass scale of a few hundred $\mathrm{MeV}$.

The relation given in Eq.(5.3) is true both perturbatively and non-perturbatively. In order to realize it non-perturbatively, let us first set

$$
\widetilde{m}^{2}\left(q^{2}\right)=m^{2}\left(q^{2}\right) H^{-1}\left(q^{2}\right),
$$

where $m^{2}\left(q^{2}\right)$ is assumed to be a RG-invariant quantity, to be identified with the dynamical gluon mass. Then

$$
\Delta\left(q^{2}\right)=\frac{H\left(q^{2}\right)}{q^{2}+m^{2}\left(q^{2}\right)},
$$

and from the requirement that $g^{2} \Delta\left(q^{2}\right)$ must be RG-invariant we have that

$$
g^{2} H\left(q^{2}\right)=\bar{g}^{2}\left(q^{2}\right) .
$$

Therefore, we finally arrive at the RG-invariant combination

$$
\widehat{d}\left(q^{2}\right) \equiv g^{2} \Delta\left(q^{2}\right)=\bar{g}^{2}\left(q^{2}\right) \bar{\Delta}\left(q^{2}\right),
$$

with

$$
\bar{\Delta}\left(q^{2}\right)=\frac{1}{q^{2}+m^{2}\left(q^{2}\right)} .
$$


Evidently the dimensionful RG-invariant quantity $\widehat{d}\left(q^{2}\right)$ is decomposed into the product of two individually RG-invariant quantities, the dimensionful part $\left[q^{2}+m^{2}\left(q^{2}\right)\right]^{-1}$ corresponding to a massive propagator (with a running mass), and the dimensionless $\bar{g}^{2}\left(q^{2}\right)$ corresponding to the running coupling (effective charge).

\section{B. The equation for the effective charge}

Even though in principle the analysis may be carried out using systematically the formulas of Appendix (A) without imposing any additional constraints on $\Delta$, the presence of the derivatives makes the numerical treatment rather cumbersome. Instead, as shown in Appendix (B) , the use of the spectral representation for $\Delta$ results in a spectacular simplification.

Specifically, assuming that $\Delta$ can be written as in (B7), and using the expressions in (B11), we have that

$$
-i \Pi(q)=\frac{(7 d-8)}{16 \pi^{2}} q^{2}\left[\int_{0}^{q^{2} / 4} d z\left(1-\frac{4 z}{q^{2}}\right)^{1 / 2} \Delta(z)-\mathcal{C}\right]+\frac{4 d}{16 \pi^{2}} \int_{0}^{q^{2} / 4} d z z\left(1-\frac{4 z}{q^{2}}\right)^{1 / 2} \Delta(z)
$$

The equation for the effective charge, $\alpha\left(q^{2}\right)=\bar{g}^{2}\left(q^{2}\right) / 4 \pi$, will be derived from (4.22) after substitution of (5.11). At this point we go to Euclidean momenta; specifically we set $q^{2}=-q_{E}^{2}$, with $q_{E}^{2}>0$ the positive square of a Euclidean four-vector, and define the Euclidean propagator as $\Delta_{E}\left(q_{E}^{2}\right)=-\Delta\left(-q_{E}^{2}\right)$ (we suppress the subscript "E" in what follows). Then, from (4.22) we have

$$
H^{-1}\left(q^{2}\right)=K+\tilde{b} g^{2}\left[\int_{0}^{q^{2} / 4} d z\left(1+\frac{4 z}{5 q^{2}}\right)\left(1-\frac{4 z}{q^{2}}\right)^{1 / 2} \Delta(z)-\mathcal{C}\right]
$$

where $\tilde{b}=10 C_{\mathrm{A}} / 48 \pi^{2}$; the discrepancy from the correct factor $b=11 C_{\mathrm{A}} / 48 \pi^{2}$, namely the first coefficient of the QCD one-loop $\beta$-function, is due to the (gauge-invariant!) omission of the ghost loops. The (infinite) constant $K$ is the gluon wave-function renormalization, introduced in order to make the equation finite, i.e. eliminate the infinite constant $\mathcal{C}$. Note that, as is typical in this type of SDE analysis, the renormalization is carried out subtractively instead of multiplicatively. This is ultimately connected with the fact that the transverse part of the three-gluon vertex is undetermined by the gauge technique (see discussion in the previous section). 
The constant $K$ may be determined from (5.12) by imposing a renormalization condition on the function $H^{-1}\left(q^{2}\right)$. Specifically, using the MOM-type of condition $H^{-1}\left(\mu^{2}\right)=1$, we have that $K$ is given by

$$
K=1-\tilde{b} g^{2}\left[\int_{0}^{\mu^{2} / 4} d z\left(1+\frac{4 z}{5 \mu^{2}}\right)\left(1-\frac{4 z}{\mu^{2}}\right)^{1 / 2} \Delta(z)-\mathcal{C}\right]
$$

Inserting the expression for $K$ given in (5.13) back into (5.12), we obtain the renormalized equation

$$
H^{-1}\left(q^{2}\right)=1+\tilde{b} g^{2}\left[\int_{0}^{q^{2} / 4} d z\left(1+\frac{4 z}{5 q^{2}}\right)\left(1-\frac{4 z}{q^{2}}\right)^{1 / 2} \Delta(z)-\int_{0}^{\mu^{2} / 4} d z\left(1+\frac{4 z}{5 \mu^{2}}\right)\left(1-\frac{4 z}{\mu^{2}}\right)^{1 / 2} \Delta(z)\right]
$$

In order to derive the equation for the effective charge $\alpha\left(q^{2}\right)=\bar{g}^{2}\left(q^{2}\right) / 4 \pi$, use the relation between $H\left(q^{2}\right)$ and $\bar{g}^{2}\left(q^{2}\right)$ given in (5.8), to cast (5.14) in the form

$\frac{1}{\bar{g}^{2}\left(q^{2}\right)}=\frac{1}{\bar{g}^{2}\left(\mu^{2}\right)}+\tilde{b}\left[\int_{0}^{q^{2} / 4} d z\left(1+\frac{4 z}{5 q^{2}}\right)\left(1-\frac{4 z}{q^{2}}\right)^{1 / 2} \Delta(z)-\int_{0}^{\mu^{2} / 4} d z\left(1+\frac{4 z}{5 \mu^{2}}\right)\left(1-\frac{4 z}{\mu^{2}}\right)^{1 / 2} \Delta(z)\right]$.

Note that again, because of the mishandling of the transverse part of the three-gluon vertex, the rhs of (5.15) is not RG-invariant. The simplest way to remedy this (by hand) is to replace $\Delta(z) \rightarrow \bar{\Delta}(z)$ of Eq.(5.10). Thus, we arrive at

$\frac{1}{\bar{g}^{2}\left(q^{2}\right)}=\frac{1}{\bar{g}^{2}\left(\mu^{2}\right)}+\tilde{b}\left[\int_{0}^{q^{2} / 4} d z\left(1+\frac{4 z}{5 q^{2}}\right)\left(1-\frac{4 z}{q^{2}}\right)^{1 / 2} \bar{\Delta}(z)-\int_{0}^{\mu^{2} / 4} d z\left(1+\frac{4 z}{5 \mu^{2}}\right)\left(1-\frac{4 z}{\mu^{2}}\right)^{1 / 2} \bar{\Delta}(z)\right]$.

Note that

$$
\frac{1}{\bar{g}^{2}(0)}=\frac{1}{\bar{g}^{2}\left(\mu^{2}\right)}-\tilde{b}\left[\int_{0}^{\mu^{2} / 4} d z\left(1+\frac{4 z}{5 \mu^{2}}\right)\left(1-\frac{4 z}{\mu^{2}}\right)^{1 / 2} \bar{\Delta}(z)\right] .
$$

\section{The equation for the gluon mass}

Let us now turn to the dynamical equation governing the evolution of the mass. From (4.23) we obtain

$$
\widetilde{m}^{2}\left(q^{2}\right)=\frac{2}{5} \tilde{b} g^{2}\left[I_{1}\left(q^{2}\right)+I_{2}\left(q^{2}\right)+I_{3}\left(q^{2}\right)+I_{4}\left(q^{2}\right)\right]
$$

where the terms $I_{i}(q)$ are given in (다). According to the discussion in (드), the terms $I_{2}\left(q^{2}\right)$ and $I_{4}\left(q^{2}\right)$ are subleading both the IR and the UV, and may be therefore safely neglected 
to a first approximation. Then, keeping only $I_{1}\left(q^{2}\right)$ and $I_{3}\left(q^{2}\right)$, we have

$$
\widetilde{m}^{2}\left(q^{2}\right)=\frac{2}{5} \tilde{b} g^{2}\left[\Delta\left(q^{2}\right) \int_{0}^{q^{2}} d y y \widetilde{m}^{2}(y) \Delta(y)-\frac{1}{2} \int_{q^{2}}^{\infty} d y y^{2} \Delta^{2}(y)\left[\widetilde{m}^{2}(y)\right]^{\prime}\right] .
$$

The next step is to rewrite this equation in terms of the RG-invariant quantities. Using (5.6) $-(5.8)$, we have that

$$
\frac{m^{2}\left(q^{2}\right)}{\bar{g}^{2}\left(q^{2}\right)}=\frac{2}{5} \tilde{b} g^{2}\left[\Delta\left(q^{2}\right) \int_{0}^{q^{2}} d y y \Delta(y)\left[m^{2}(y) / \bar{g}^{2}(y)\right]-\frac{1}{2} \int_{q^{2}}^{\infty} d y y^{2} \Delta^{2}(y)\left[m^{2}(y) / \bar{g}^{2}(y)\right]^{\prime}\right]
$$

Given that $\bar{g}^{2}(y)$ is expected to be a much slower varying function of the momentum compared to $m^{2}(y)$, both in the UV and the IR, we will simplify the analysis by neglecting the derivative $\left[\bar{g}^{2}(y)\right]^{\prime}$ next to $\left[m^{2}(y)\right]^{\prime}$. Then, we have that

$\frac{m^{2}\left(q^{2}\right)}{\bar{g}^{2}\left(q^{2}\right)}=\frac{2 \tilde{b}}{5}\left[\Delta\left(q^{2}\right) \int_{0}^{q^{2}} d y y m^{2}(y)\left\{g^{2} \Delta(y) / \bar{g}^{2}(y)\right\}-\frac{1}{2} \int_{q^{2}}^{\infty} d y y^{2}\left[m^{2}(y)\right]^{\prime} \Delta(y)\left\{g^{2} \Delta(y) / \bar{g}^{2}(y)\right\}\right]$,

which, after using (5.9), becomes

$$
\frac{m^{2}\left(q^{2}\right)}{\bar{g}^{2}\left(q^{2}\right)}=\frac{2 \tilde{b}}{5}\left[\Delta\left(q^{2}\right) \int_{0}^{q^{2}} d y y m^{2}(y) \bar{\Delta}(y)-\frac{1}{2} \int_{q^{2}}^{\infty} d y y^{2}\left[m^{2}(y)\right]^{\prime} \Delta(y) \bar{\Delta}(y)\right] .
$$

Finally, the rhs of (15.22) is made RG-invariant by setting $\Delta\left(q^{2}\right) \rightarrow \bar{\Delta}\left(q^{2}\right)$ and $\Delta(y) \rightarrow \widehat{d}(y)$, thus obtaining

$$
\frac{m^{2}\left(q^{2}\right)}{\bar{g}^{2}\left(q^{2}\right)}=\frac{2 \tilde{b}}{5}\left[\bar{\Delta}\left(q^{2}\right) \int_{0}^{q^{2}} d y y m^{2}(y) \bar{\Delta}(y)-\frac{1}{2} \int_{q^{2}}^{\infty} d y y^{2} \bar{\Delta}^{2}(y) \bar{g}^{2}(y)\left[m^{2}(y)\right]^{\prime}\right] .
$$

Let us now study the behavior of the solutions of (15.23) for asymptotically large $q^{2}$; in this limit we set $\bar{\Delta}(x) \rightarrow 1 / x$ and $\bar{\Delta}(y) \rightarrow 1 / y$. Then, the equation reduces to

$$
m^{2}\left(q^{2}\right) \ln q^{2}=\frac{2}{5}\left[\frac{1}{q^{2}} \int_{0}^{q^{2}} d y m^{2}(y)-\frac{1}{2} \int_{q^{2}}^{\infty} d y \bar{g}^{2}(y)\left[m^{2}(y)\right]^{\prime}\right] .
$$

It is relatively straightforward to establish that the asymptotic solutions of (5.24) display power-law running. Indeed, substituting on both sides of (5.24) a $\mathrm{m}^{2}\left(q^{2}\right)$ of the form

$$
m^{2}\left(q^{2}\right)=\frac{\lambda_{0}^{4}}{q^{2}}\left(\ln q^{2}\right)^{\gamma-1}
$$

it is easy to recognize that the second term on the rhs of (5.25) is subleading. Indeed, in the absence of $\bar{g}^{2}(y)=(b \ln y)^{-1}$ the integrand is a total derivative, which yields to the rhs simply a term $\frac{1}{2} m^{2}\left(q^{2}\right)$; this is suppressed, because it is not multiplied by a $\ln q^{2}$. The 
presence of $\bar{g}^{2}(y)$ suppresses this integral even further. Specifically, integration by parts and

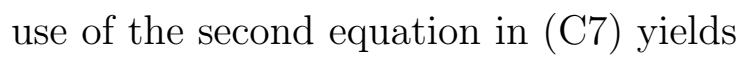

$$
\int_{q^{2}}^{\infty} d y \bar{g}^{2}(y)\left[m^{2}(y)\right]^{\prime}=-m^{2}(x) \bar{g}^{2}(x)+\mathcal{O}(1 / \ln x),
$$

which is indeed further suppressed by an extra logarithm.

Thus, using the elementary integral

$$
\int \frac{d y}{y(\ln y)^{1+a}}=-\frac{1}{a(\ln y)^{a}}
$$

[first equation in (C7)], we have that (5.25) is a solution of (5.24) provided that

$$
\gamma=\frac{2}{5}
$$

and so, the asymptotic solution has power-law running, given by

$$
m^{2}\left(q^{2}\right)=\frac{\lambda_{0}^{4}}{q^{2}}\left(\ln q^{2}\right)^{-3 / 5}
$$

Finally, if we were to assume the approximate validity of (5.29) for the entire range of momenta, we can set $\left[m^{2}(y)\right]^{\prime} \approx-m^{2}(y) / y$; that way, we convert (5.24) from an integrodifferential equation to the simpler integral equation

$$
\frac{m^{2}\left(q^{2}\right)}{\bar{g}^{2}\left(q^{2}\right)}=\frac{2 \tilde{b}}{5}\left[\bar{\Delta}\left(q^{2}\right) \int_{0}^{q^{2}} d y y m^{2}(y) \bar{\Delta}(y)+\frac{1}{2} \int_{q^{2}}^{\infty} d y y \bar{\Delta}^{2}(y) \bar{g}^{2}(y) m^{2}(y)\right] .
$$

\section{Solving the system numerically}

We will next discuss the numerical solutions for the system of integral equations, namely (5.16) and (5.30) coupled together.

We solve numerically the two coupled integral equations, renormalizing them at three different points, namely $\mu=\{4,10,91\} \mathrm{GeV}$, with $\alpha\left(\mu^{2}\right)=g^{2}(\mu) / 4 \pi=\{0.341,0.229,0.127\}$, respectively. In Fig. 6, we show the results for $\alpha\left(q^{2}\right)$; there we see clearly that the three curves merge practically into a single one, thus confirming numerically the $\mu$-independence

of $\alpha\left(q^{2}\right)$, expected on formal grounds. These three curves may be accurately fitted by the physically motivated functional form [1], namely

$$
\alpha\left(q^{2}\right)=\frac{1}{4 \pi \tilde{b} \ln \left[\left(q^{2}+t m_{0}^{2}\right) / \Lambda^{2}\right]},
$$




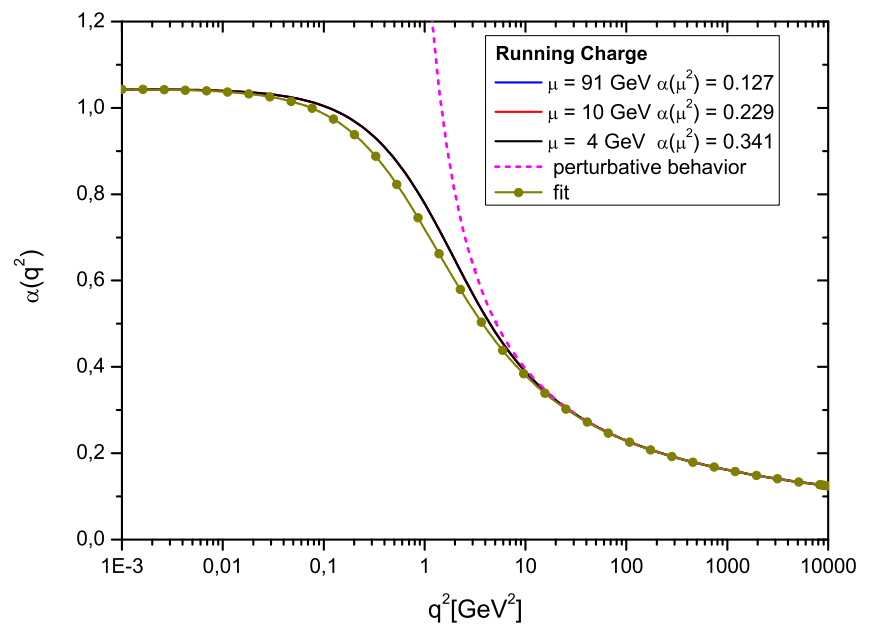

FIG. 6: Numerical solutions for the effective charge obtained from Eq. (5.16), renormalized at three different points: $\mu=4 \mathrm{GeV}$ and $\alpha\left(\mu^{2}\right)=0.341$ (black curve), $\mu=10 \mathrm{GeV}$ and $\alpha\left(\mu^{2}\right)=0.229$ (red curve), $\mu=91 \mathrm{GeV}$ and $\alpha\left(\mu^{2}\right)=0.127$ (blue curve). The three curves practically coincide, showing that indeed $\alpha\left(q^{2}\right)$ is independent of the renormalization point chosen. The dashed curve (magenta) is the perturbative one-loop behavior, and the brown line with circles depicts the fit of Eq.(5.31), for $t=3.7$.

with $t=3.7$ and $\Lambda=645 \mathrm{MeV}$ [see caption of Fig. 6].

In Fig. 7, we show the dynamical gluon mass, $m^{2}\left(q^{2}\right)$, obtained as solution of Eq. (5.30) at the same renormalization points of Fig. 6. Once again, this figure shows us that $m^{2}\left(q^{2}\right)$ is also a RG-invariant quantity, since the three curves, obtained using the three different (and quite disparate) renormalization points, are practically on top of each other. The behavior of $m^{2}\left(q^{2}\right)$ in the entire range of momenta can be accurately described by the following parametrization

$$
m^{2}\left(q^{2}\right)=\frac{m_{0}^{4}}{q^{2}+m_{0}^{2}}\left[\ln \left(\frac{q^{2}+f\left(q^{2}, m_{0}^{2}\right)}{\Lambda^{2}}\right) / \ln \left(\frac{f\left(0, m_{0}^{2}\right)}{\Lambda^{2}}\right)\right]^{-3 / 5},
$$

where the function

$$
f\left(q^{2}, m_{0}^{2}\right)=\rho_{1} m_{0}^{2}+\rho_{2} \frac{m_{0}^{4}}{q^{2}+m_{0}^{2}},
$$

with $\rho_{1}=-1 / 2, \rho_{2}=5 / 2$, and $m_{0}=612 \mathrm{MeV}$. Notice that in the UV asymptotic limit the above expression goes over to that of Eq.(5.29), as it should.

Finally, we turn to the RG-invariant quantity $\widehat{d}\left(q^{2}\right)$, which appears in a natural way in 


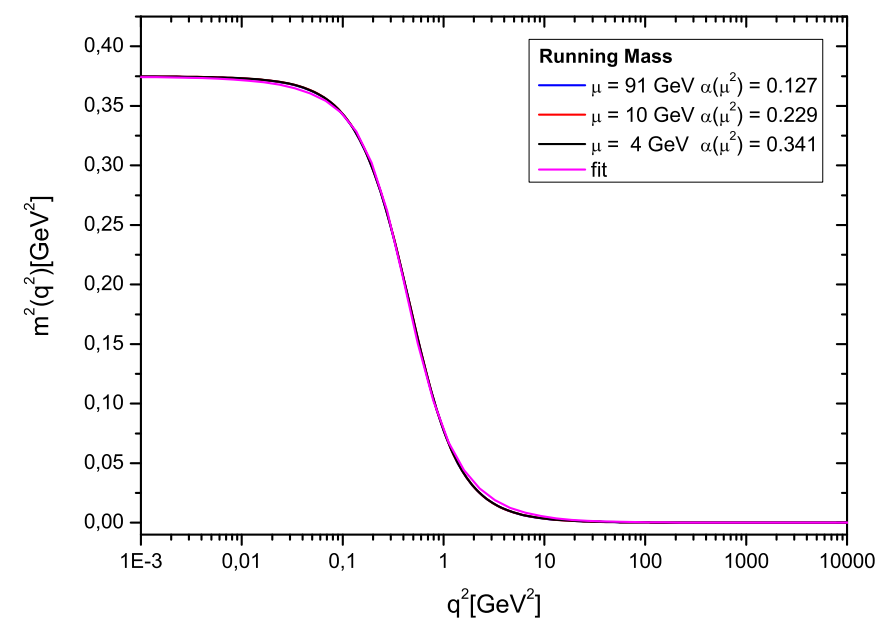

FIG. 7: The effective gluon mass, $m^{2}\left(q^{2}\right)$, for the same renormalization points used in Fig. (66). Evidently, the three curves merge into a single one, showing that the numerical solutions are independent of the renormalization point. The continuous line in magenta is the fit of Eq. (5.32) with $\rho_{1}=-1 / 2$ and $\rho_{2}=5 / 2$.

all physical process involving gluon exchange. With the help of Eq. (5.9) we can construct $\widehat{d}\left(q^{2}\right)$ out of the numerical solutions for $\alpha\left(q^{2}\right)$ and $m^{2}\left(q^{2}\right)$; the result is shown in Fig. 8, Obviously, since $\widehat{d}\left(q^{2}\right)$ is built out of two quantities that are individually independent of $\mu$, it too turns out to be $\mu$-independent; this property is clearly observed in Fig. 8 .

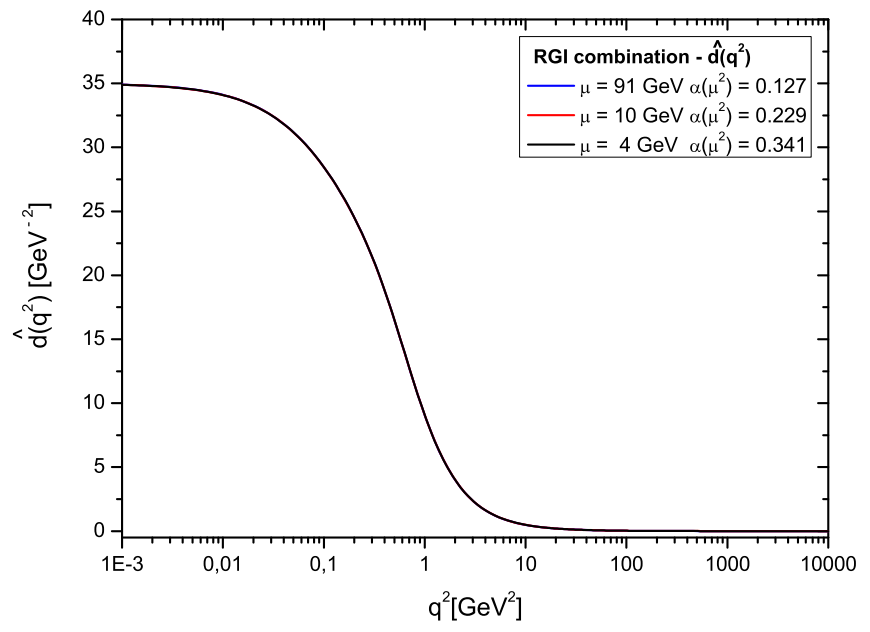

FIG. 8: The renormalization-group invariant product $\widehat{d}\left(q^{2}\right)$ obtained by combining the results for $\alpha\left(q^{2}\right)$ and $m^{2}\left(q^{2}\right)$ according to Eq. (5.9). 


\section{DISCUSSION AND CONCLUSIONS}

In this article we have demonstrated how to obtain a finite gluon mass from the SDE of QCD, formulated in the PT-BFM framework. Obtaining a finite mass without the appearance of seagull divergences has been a long-standing problem, that has afflicted all related studies for a number of years. The key observation that leads to the solution of this problem is that a judicious Ansatz for the three-gluon vertex eliminates all seagull divergences by means of a basic identity, valid in dimensional regularization. In retrospect one realizes that the problem of seagull divergences is not intrinsic to this approach, but has rather been caused by the inadvertent mismatch of two field theoretic mechanisms, induced by an imperfect Ansatz for the vertex. Specifically, the Schwinger mechanism, which requires the appearance of massless poles in the three-gluon vertex, distorts the mechanism responsible for the cancellation of the seagull divergences, unless the poles enter into the gluon vertex in a very particular way.

The procedure described in the present work furnishes eventually two separate but coupled equations for the QCD effective charge and the gluon mass, which, when solved simultaneously, yield a unique answer for both quantities. This is a considerable improvement over the existing approaches (e.g. [1, 10, 11]) where one had only one dynamical equation, determining $\widehat{d}(q)$, which was subsequently decomposed according to Eq. (5.9), in order to obtain (not without a certain ambiguity) the effective charge and gluon mass. It should be emphasized, however, that even though the values obtained for $\alpha(0)$ and $m_{0}$ are very reasonable, they are not directly comparable with the values obtained from phenomenological studies, due to the fact that the gauge used in this work ("stagnant" or "generalized Feynman") is not the canonical Feynman gauge of the BFM, which, as is well-known, furnishes the PT effective charge and gluon mass. This may account, in retrospect, for the slightly elevated value of $\alpha(0) \approx 1$ obtained here, compared to a value of $0.5-0.7$, found in recent theoretical analysis [52], and various phenomenological studies [53, 54]

The fully dressed three-gluon vertex $\widetilde{\Gamma}_{\mu \alpha \beta}$ used in Eq. (4.6) satisfies (by construction) the WI given in Eq. (4.3), or, after restoring the longitudinal terms, the full WI of Eq. (2.6), which is crucial for ensuring the transversality of the gluon self-energy. However, this Ansatz used for $\widetilde{\Gamma}_{\mu \alpha \beta}$ is still incomplete from the point of view of full Bose symmetry, in the sense that it does not satisfy the correct STI when contracted with respect to the two other legs; 
we remind the reader that the other two legs (internal lines, irrigated by virtual momenta) correspond to "quantum" gluons, as opposed to the external (background) gluon, where the physical momentum $q$ enters. The corresponding STI satisfied when contracting $\widetilde{\Gamma}_{\mu \alpha \beta}$ with respect to the quantum legs is a variant of the well-known STI satisfied by the conventional vertex [55], and has been derived in [47]. The construction of a three-gluon vertex satisfying the correct WI and STIs is currently under investigation, and we hope to report the results in the near future.

An additional technical issue related to the form of $\widetilde{\Gamma}_{\mu \alpha \beta}$ is the omission of the identically conserved part, as already mentioned in the corresponding sections. It would be most interesting to extend the QED construction of [56, 57] to the case of the three-gluon vertex $\widetilde{\Gamma}_{\mu \alpha \beta}$. Such a task, however, appears to be of formidable logistic complexity, given that there are thirteen linearly independent tensorial structures, with their corresponding formfactors. The techniques and the special tensorial bases introduced in [58] may prove useful for simplifying such a task.

In a similar spirit, the part $V^{\alpha \beta \gamma}$ of the vertex containing the massless poles, thus triggering the Schwinger mechanism, should also be appropriately extended, to satisfy the correct WI with respect to all three legs. A prime candidate for this role is the vertex proposed in [59], given by

$V^{\alpha \beta \gamma}\left(k_{1}, k_{2}, k_{3}\right)=\frac{k_{1}^{\alpha} k_{2}^{\beta}\left(k_{1}-k_{2}\right)^{\mu}}{2 k_{1}^{2} k_{2}^{2}} P_{\mu}^{\gamma}\left(k_{3}\right) m^{2}\left(k_{3}\right)-\frac{k_{3}^{\gamma}}{k_{3}^{2}}\left[m^{2}\left(k_{2}\right)-m^{2}\left(k_{1}\right)\right] P_{\mu}^{\alpha}\left(k_{1}\right) P^{\mu \beta}\left(k_{2}\right)+\mathrm{cp}$,

where "cp" denotes "cyclic permutations". Note that a vertex such as (6.1) will furnish a concrete (but still not unique) expression for $V^{\mathrm{t}}$, which, in turn, will allow one to scrutinize some of the assumptions made in Section IV. Of course, for self-consistency, one should perform the analysis in, e.g., the Landau or conventional Feynman gauges, rather then the "generalized" Feynman gauge employed here; calculations in this direction are already in progress. 


\section{APPENDIX A: SOME USEFUL RELATIONS}

Let us define the following quantities,

$$
\begin{aligned}
R_{f}(q) & \equiv \int_{k} \frac{f(k+q)-f(k)}{(k+q)^{2}-k^{2}}, \\
T_{f}(q) & \equiv \int_{k} k^{2} \frac{f(k+q)-f(k)}{(k+q)^{2}-k^{2}}+\frac{d}{2} \int_{k} f(k),
\end{aligned}
$$

for an arbitrary function $f(x)$ that is finite at the origin.

Let us define $q^{2}=x, k^{2}=y,(k+q)^{2}=z$, and let us write the (Euclidean) integration measure $\left[d^{d} k=i d^{d} k_{E}\right]$ in spherical coordinates

$$
\int d^{d} k_{E}=2 \pi \int_{0}^{\pi} d \theta(\sin \theta)^{d-2} \int_{0}^{\infty} d y y .
$$

We then have that $z=y+x+2 \sqrt{x y} \cos \theta$, and we define $w \equiv z-y=x+2 \sqrt{x y} \cos \theta$. Finally, recall the elementary integral

$$
\int_{0}^{\pi} d \theta \sin ^{m} \theta \cos ^{n} \theta= \begin{cases}\frac{\Gamma\left(\frac{m+1}{2}\right) \Gamma\left(\frac{n+1}{2}\right)}{\Gamma\left(\frac{m+n+2}{2}\right)}, & n=2 k \\ 0, & n=2 k+1\end{cases}
$$

$R_{f}(q)$ and $T_{f}(q)$ may be expanded systematically as a power series in $q^{2}$. To that end we

consider the Taylor expansion of $f(z)$ around $w=0$, which gives (we are assuming finite derivatives at the origin),

$$
\frac{f(z)-f(y)}{w}=f^{\prime}(y)+\frac{w}{2 !} f^{\prime \prime}(y)+\frac{w^{2}}{3 !} f^{\prime \prime \prime}(y)+\ldots
$$

where the primes denote differentiations with respect to $y$. Then, one must collect pieces of a given order in $q^{2}$ from the various powers of $w$, using (A33).

It is clear, for example, that when the term $f^{\prime}(y)$ on the rhs of (A4 $)$ is inserted into $T_{f}(q)$ generates the seagull identity (3.14), while all remaining terms proportional to positive powers of $w$; so,

$$
T_{f}(0)=0
$$

As a second example, we determine the term of $R_{f}(q)$ linear in $q^{2}$, to be denoted by $R_{f}^{(1)}(q)$; to accomplish this one must collect the appropriate contributions coming from both the second and the third term on the rhs of (A4). Using (3.14 $)$ one then obtains

$$
R_{f}^{(1)}(q)=q^{2} \int_{k}\left[\frac{1}{2} f^{\prime \prime}\left(k^{2}\right)+\frac{1}{6} k^{2} f^{\prime \prime \prime}\left(k^{2}\right)\right]
$$


or after partial integration, assuming that $\left[y f^{\prime}\right]_{0}^{\infty}=0$ and $\left[y^{2} f^{\prime \prime}\right]_{0}^{\infty}=0$ (valid when $f(0)$ is finite, and $f(y) \sim y$ (or faster) at infinity), we have

$$
R_{f}^{(1)}(q)=\frac{c}{6} q^{2} f(0)
$$

where $c \equiv i / 16 \pi^{2}$.

\section{APPENDIX B: THE SPECTRAL REPRESENTATION}

Let us consider a simple massive tree-level propagator,

$$
d_{m}(q)=\frac{1}{q^{2}-m^{2}}
$$

and set $f=d_{m}$ directly into (A1). Turns out that both $R_{d_{m}}(q)$ and $T_{d_{m}}(q)$ can be calculated exactly; specifically, using that

$$
\frac{d_{m}(k+q)-d_{m}(k)}{(k+q)^{2}-k^{2}}=-d_{m}(k) d_{m}(k+q),
$$

it is elementary to show that

$$
R_{d_{m}}(q)=c\left[\int_{0}^{1} d x \ln \left(1+\frac{q^{2} x(x-1)}{m^{2}}\right)-C_{m}\right],
$$

where the cutoff-dependent constant $C_{m}$ is given in dimensional regularization by

$$
C_{m}=\frac{2}{\epsilon}-\gamma-\ln \left(\frac{m^{2}}{4 \pi \mu^{2}}\right)
$$

Evidently, $R_{d_{m}}(0)=-c C_{m}$. As an additional check, note that the term of $R_{d_{m}}(q)$ linear in $q^{2}$, obtained by Taylor-expanding ( $(\bar{B} 3)$, is given by

$$
R_{d_{m}}^{(1)}(q)=-\frac{c}{6} \frac{q^{2}}{m^{2}}
$$

which coincides with the result obtained when substituting $f(0)=d_{m}(0)=-\frac{1}{m^{2}}$ in the general formula of (A).

For $T_{d_{m}}(q)$ we have, using the identities (3.6)-(3.7),

$$
\begin{aligned}
T_{d_{m}}(q) & =m^{2} \int_{k} \frac{q^{2}+2 q \cdot k}{\left(k^{2}-m^{2}\right)^{2}\left[(k+q)^{2}-m^{2}\right]} \\
& =-c m^{2} q^{2} \int_{0}^{1} d x \frac{x(2 x-1)}{q^{2} x(1-x)+m^{2}} \\
& =c m^{2} \int_{0}^{1} d x \ln \left(1+\frac{q^{2} x(x-1)}{m^{2}}\right) .
\end{aligned}
$$


Evidently, $T_{d_{m}}(0)=0$, in agreement with (A5).

The results of (B3)-(B6) may be used in a more general way. Specifically, if we assume a spectral representation for the gluon propagator [1, 60], namely

$$
\Delta\left(q^{2}\right)=\int d \lambda^{2} \frac{\rho\left(\lambda^{2}\right)}{q^{2}-\lambda^{2}+i \epsilon}
$$

then from (푸) (with $m \rightarrow \lambda$ ) we have

$$
\begin{aligned}
& R_{\Delta}(q)=c\left[\int d \lambda^{2} \rho\left(\lambda^{2}\right) \int_{0}^{1} d x \ln \left(1+\frac{q^{2} x(x-1)}{\lambda^{2}}\right)-\mathcal{C}\right] \\
& T_{\Delta}(q)=c \int d \lambda^{2} \rho\left(\lambda^{2}\right) \lambda^{2} \int_{0}^{1} d x \ln \left(1+\frac{q^{2} x(x-1)}{\lambda^{2}}\right)
\end{aligned}
$$

where

$$
\mathcal{C}=\int d \lambda^{2} \rho\left(\lambda^{2}\right) C_{\lambda}
$$

Note that after the renormalization of the corresponding $\mathrm{SDE}$ (see Section $\mathrm{V}$ ) $\mathcal{C}$ will eventually drop out. Then, the use of the following identities [61]

$$
\begin{aligned}
\int d \lambda^{2} \rho\left(\lambda^{2}\right) \int_{0}^{1} d x \ln \left(1+\frac{q^{2} x(x-1)}{\lambda^{2}}\right) & =\int_{0}^{q^{2} / 4} d z\left(1-\frac{4 z}{q^{2}}\right)^{1 / 2} \Delta(z) \\
\int d \lambda^{2} \rho\left(\lambda^{2}\right) \lambda^{2} \int_{0}^{1} d x \ln \left(1+\frac{q^{2} x(x-1)}{\lambda^{2}}\right) & =\int_{0}^{q^{2} / 4} d z z\left(1-\frac{4 z}{q^{2}}\right)^{1 / 2} \Delta(z),
\end{aligned}
$$

allows one to cast $R_{\Delta}(q)$ and $T_{\Delta}(q)$ again as an integral containing the gluon propagator $\Delta$, namely

$$
\begin{aligned}
& R_{\Delta}(q)=c\left[\int_{0}^{q^{2} / 4} d z\left(1-\frac{4 z}{q^{2}}\right)^{1 / 2} \Delta(z)-\mathcal{C}\right], \\
& T_{\Delta}(q)=c \int_{0}^{q^{2} / 4} d z z\left(1-\frac{4 z}{q^{2}}\right)^{1 / 2} \Delta(z) .
\end{aligned}
$$

Note that the simple change of variables $t=4 z / q^{2}$ allows one to cast $R_{\Delta}(q)$ and $T_{\Delta}(q)$ in the alternative form

$$
\begin{aligned}
& R_{\Delta}(q)=c\left[\left(q^{2} / 4\right) \int_{0}^{1} d t(1-t)^{1 / 2} \Delta\left(t q^{2} / 4\right)-\mathcal{C}\right] \\
& T_{\Delta}(q)=c\left(q^{2} / 4\right)^{2} \int_{0}^{1} d t t(1-t)^{1 / 2} \Delta\left(t q^{2} / 4\right)
\end{aligned}
$$

which makes the identification of the IR behavior of these quantities immediate, and is particularly useful for their numerical treatment. 


\section{APPENDIX C: THE MASS EQUATION}

Let us consider (in Euclidean space) the integral appearing on the rhs of (4.21), to be denoted by $I(q)$. We have

$$
I(q)=\frac{1}{q^{2}} \int_{k_{E}} \widetilde{m}^{2}(k) \Delta(k) \Delta(k+q)\left[(k+q)^{2}-k^{2}\right],
$$

which, with the notation introduced in Appendix ( $(\underline{\mathrm{A}})$, reads

$$
I(x)=\int_{k_{E}} \widetilde{m}^{2}(y) \Delta(y) \Delta(z)\left[1+\frac{2 \sqrt{y}}{\sqrt{x}} \cos \theta\right] .
$$

Then, expand $\Delta(z)=\Delta(y)+w \Delta^{\prime}(y)+\ldots$, and collect the terms that survive the angular integration, to obtain

$$
\begin{aligned}
I(0) & =\int_{k_{E}} \widetilde{m}^{2}\left(k^{2}\right) \Delta\left(k^{2}\right)\left[\Delta\left(k^{2}\right)+4 k^{2} \Delta^{\prime}\left(k^{2}\right) \cos ^{2} \theta\right] \\
& =\int_{k_{E}} \widetilde{m}^{2}\left(k^{2}\right) \Delta\left(k^{2}\right)\left[\Delta\left(k^{2}\right)+k^{2} \Delta^{\prime}\left(k^{2}\right)\right] \\
& =-\frac{1}{2} \int_{k_{E}} k^{2} \Delta^{2}\left(k^{2}\right)\left[\widetilde{m}^{2}\left(k^{2}\right)\right]^{\prime}
\end{aligned}
$$

Note that a monotonically decreasing mass, $\left[\widetilde{m}^{2}\left(k^{2}\right)\right]^{\prime}<0$, guarantees that $I(0)>0$, or, equivalently, the positivity of $\widetilde{m}^{2}(0)$ in Euclidean space.

To write $I(x)$ in a form suitable for solving the corresponding dynamical equation, first split the radial integration into two intervals, $\int_{0}^{\infty} d y=\int_{0}^{x} d y+\int_{x}^{\infty} d y$; in the first interval apply the usual approximation

$$
\int_{0}^{x} d y f_{1}(z) f_{2}(y) \approx f_{1}(x) \int_{0}^{x} d y f_{2}(y)
$$

while in the second, since $x<y$, we can carry out the Taylor expansion as before. Thus, we obtain

$$
I(x) \approx I_{1}(x)+I_{2}(x)+I_{3}(x)+I_{4}(x),
$$

with

$$
\begin{aligned}
& I_{1}(x)=\Delta(x) \int_{0}^{x} d y y \widetilde{m}^{2}(y) \Delta(y) \\
& I_{2}(x)=-\frac{\Delta(x)}{x} \int_{0}^{x} d y y^{2} \widetilde{m}^{2}(y) \Delta(y) \\
& I_{3}(x)=-\frac{1}{2} \int_{x}^{\infty} d y y^{2} \Delta^{2}(y)\left[\widetilde{m}^{2}(y)\right]^{\prime} \\
& I_{4}(x)=-\frac{1}{2} \widetilde{m}^{2}(x) x^{2} \Delta^{2}(x) .
\end{aligned}
$$


Note that, as $x \rightarrow 0, I_{1}(x), I_{2}(x)$ and $I_{4}(x)$ vanish, and one recovers from $I_{3}(x)$ the exact result for $I(0)$ given in (C3) .

Finally, note that for a $m^{2}(x)$ displaying the asymptotic behavior given in (5.25) the following results are useful [11],

$$
\begin{aligned}
\frac{1}{x} \int_{0}^{x} d y m^{2}(y) & =\gamma^{-1} m^{2}(x) \ln x+\frac{c^{\prime}}{x}, \\
\int_{x}^{\infty} d y \frac{m^{2}(y)}{y} & =m^{2}(x)+\mathcal{O}(1 / \ln x), \\
\frac{1}{x^{2}} \int_{0}^{x} d y y m^{2}(y) & =m^{2}(x)+\mathcal{O}(1 / \ln x),
\end{aligned}
$$

where $c^{\prime}$ is a constant. The first equation is derived using directly the integral of (5.27), while for the other two we have employed the asymptotic property of the incomplete $\Gamma(a, u)$ function. Specifically,

$$
\Gamma(a, u)=\int_{u}^{\infty} d t e^{-t} t^{a-1},
$$

(with no restriction on the sign of $a$ ), and its asymptotic representation for large values of $|u|$ is given by

$$
\Gamma(a, u)=u^{a-1} e^{-u}+\mathcal{O}\left(|u|^{-1}\right) .
$$

\section{ACKNOWLEDGMENTS}

The research of J. P. is supported by the European FEDER and Spanish MICINN under grant FPA2008-02878, and the Fundación General of the UV.

[1] J. M. Cornwall, Phys. Rev. D 26, 1453 (1982).

[2] G. Parisi and R. Petronzio, Phys. Lett. B 94, 51 (1980); C. W. Bernard, Phys. Lett. B 108, 431 (1982); Nucl. Phys. B 219, 341 (1983); J. F. Donoghue, Phys. Rev. D 29, 2559 (1984); J. E. Mandula and M. Ogilvie, Phys. Lett. B 185, 127 (1987); F. Halzen, G. I. Krein and A. A. Natale, Phys. Rev. D 47, 295 (1993); F. J. Yndurain, Phys. Lett. B 345 (1995) 524; A. Szczepaniak, E. S. Swanson, C. R. Ji and S. R. Cotanch, Phys. Rev. Lett. 76, 2011 (1996); J. H. Field, Phys. Rev. D 66, 013013 (2002); E. G. S. Luna, A. F. Martini, M. J. Menon, A. Mihara and A. A. Natale, Phys. Rev. D 72, 034019 (2005).

[3] F. J. Dyson, Phys. Rev. 75, 1736 (1949).

[4] J. S. Schwinger, Proc. Nat. Acad. Sci. 37, 452 (1951); Proc. Nat. Acad. Sci. 37, 455 (1951). 
[5] J. M. Cornwall and J. Papavassiliou, Phys. Rev. D 40, 3474 (1989).

[6] D. Binosi and J. Papavassiliou, Phys. Rev. D 66, 025024 (2002); Phys. Rev. D 66, 111901 (2002); J. Phys. G 30, 203 (2004).

[7] V. P. Nair, "Quantum field theory: A modern perspective," New York, USA: Springer (2005) $557 \mathrm{p}$.

[8] D. Binosi and J. Papavassiliou, Phys. Rept. 479, 1 (2009).

[9] L. F. Abbott, Nucl. Phys. B 185, 189 (1981).

[10] A. C. Aguilar and J. Papavassiliou, JHEP 0612, 012 (2006).

[11] A. C. Aguilar and J. Papavassiliou, Eur. Phys. J. A 35, 189 (2008).

[12] A. C. Aguilar, D. Binosi and J. Papavassiliou, Phys. Rev. D 78, 025010 (2008).

[13] J. S. Schwinger, Phys. Rev. 125, 397 (1962).

[14] J. S. Schwinger, Phys. Rev. 128, 2425 (1962).

[15] R. Jackiw and K. Johnson, Phys. Rev. D 8, 2386 (1973).

[16] J. M. Cornwall and R. E. Norton, Phys. Rev. D 83338 (1973).

[17] E. Eichten and F. Feinberg, Phys. Rev. D 10, 3254 (1974).

[18] R. Jackiw, "Dynamical Symmetry Breaking," In *Erice 1973, Proceedings, Laws Of Hadronic Matter*, New York 1975, 225-251 and M I T Cambridge - COO-3069-190 (73,REC.AUG 74) $23 p$.

[19] E. Farhi and R. Jackiw, "Dynamical Gauge Symmetry Breaking. A Collection Of Reprints," Singapore, Singapore: World Scientific (1982) $403 p$.

[20] P. H. Frampton, "Gauge Field Theories: Third Revised and Improved Edition," John Wiley and Sons, Inc, 2008.

[21] A. C. Aguilar, D. Binosi and J. Papavassiliou, PoS LC2008, 050 (2008).

[22] A. C. Aguilar, D. Binosi, J. Papavassiliou and J. Rodriguez-Quintero Phys. Rev. D 80, 085018 (2009).

[23] J. M. Cornwall, Nucl. Phys. B 157, 392 (1979).

[24] J. M. Cornwall, Phys. Rev. D 57, 7589 (1998).

[25] P. de Forcrand and M. D'Elia, Phys. Rev. Lett. 82, 4582 (1999).

[26] See, for example, J. Greensite, Prog. Part. Nucl. Phys. 51, 1 (2003), and references therein.

[27] J. Greensite, K. Langfeld, S. Olejnik, H. Reinhardt and T. Tok, Phys. Rev. D 75, 034501 (2007). 
[28] Conversely, as has been argued recently in S. J. Brodsky and R. Shrock, Phys. Lett. B 666, 95 (2008), confinement induces a maximum wavelength, which, in turn, can be associated with an effective gluon mass.

[29] Lattice simulations of gauge-dependent quantities are known to suffer from the problem of the Gribov copies, but it is generally believed that the effects are quantitative rather than qualitative, see, e.g., A. G. Williams, Prog. Theor. Phys. Suppl. 151, 154 (2003); A. Sternbeck et al., AIP Conf. Proc. 756, 284 (2005); P. J. Silva and O. Oliveira, Nucl. Phys. B 690, 177 (2004).

[30] C. Alexandrou, P. de Forcrand and E. Follana, Phys. Rev. D 63, 094504 (2001); F. D. R. Bonnet, P. O. Bowman, D. B. Leinweber and A. G. Williams, Phys. Rev. D 62, 051501 (2000); F. D. R. Bonnet, P. O. Bowman, D. B. Leinweber, A. G. Williams and J. M. Zanotti, Phys. Rev. D 64, 034501 (2001).

[31] A. Cucchieri and T. Mendes, PoS LAT2007, 297 (2007); Phys. Rev. Lett. 100, 241601 (2008); arXiv:0904.4033 [hep-lat].

[32] I. L. Bogolubsky et al., PoS LATTICE, 290 (2007).

[33] P. O. Bowman et al., Phys. Rev. D 76, 094505 (2007).

[34] T. Iritani, H. Suganuma and H. Iida, arXiv:0908.1311 [hep-lat].

[35] D. Dudal, J. A. Gracey, S. P. Sorella, N. Vandersickel and H. Verschelde, Phys. Rev. D 78, 065047 (2008).

[36] V. N. Gribov, Nucl. Phys. B 139, 1 (1978).

[37] D. Zwanziger, Nucl. Phys. B 412, 657 (1994).

[38] M. Lavelle, Phys. Rev. D 44, 26 (1991).

[39] J. S. Ball and T. W. Chiu, Phys. Rev. D 22, 2542 (1980).

[40] Schwinger demonstrated how his mechanism works in $\mathrm{QED}_{2}$ with massless fermions, which is exactly solvable [14]. $\Pi\left(q^{2}\right)$ does indeed have a pole at $q^{2}=0$, and the photon acquires a mass, given by $\mu^{2}=e^{2} / \pi$.

[41] In fact, the Higgs mechanism can be viewed as just a special realization of the Schwinger mechanism, where the residue of the pole is saturated by $v^{2}$, where $v$ is the vacuum expectation value of a canonical scalar field coupled to the vector meson.

[42] The demonstration of the existence of a bound state, and in particular a zero-mass bound state, in realistic field theories is a difficult dynamical problem, usually studied by means of 
integral equations known as Bethe-Salpeter equations (see, e.g., E. C. Poggio, E. Tomboulis and S. H. Tye, Phys. Rev. D 11, 2839 (1975)).

[43] An additional important step would be to demonstrate that all Goldstone-like scalars are canceled out of the $S$-matrix by other massless poles or by current conservation. This cancellation was explicitly demonstrated in a $d=4$ Abelian toy model, e.g. [15, 18], and the conditions for its realization in non-Abelian theories were studied in [17].

[44] In the presence of a hard cutoff these integrals are quadratically divergent, up to logarithmic corrections.

[45] M. E. Peskin and D. V. Schroeder, "An Introduction To Quantum Field Theory," Reading, USA: Addison-Wesley (1995) 842 p.

[46] J. C. Collins, "Renormalization". Cambridge, Uk: Univ. Pr. (1984) 380p.

[47] D. Binosi and J. Papavassiliou, JHEP 0811, 063 (2008).

[48] D. Binosi and J. Papavassiliou, Phys. Rev. D 77, 061702 (2008).

[49] This type of generalized Feynman gauge corresponds to the so-called "stagnant gauge", presented in C. H. Llewellyn Smith, Nucl. Phys. B 165, 423 (1980). It may be formally reached by introducing in the Feynman diagrams a momentum dependent $\xi\left(q^{2}\right)$, or an operator $\xi(\square)$ in the original QCD Lagrangian.

[50] P. A. Grassi, T. Hurth and M. Steinhauser, Annals Phys. 288, 197 (2001).

[51] A. C. Aguilar, D. Binosi and J. Papavassiliou, arXiv:0907.0153 [hep-ph].

[52] J. M. Cornwall, arXiv:0904.3758 [hep-ph].

[53] A. C. Aguilar, A. A. Natale and P. S. Rodrigues da Silva, Phys. Rev. Lett. 90, 152001 (2003).

[54] A. C. Aguilar, A. Mihara and A. A. Natale, Phys. Rev. D 65, 054011 (2002); A. C. Aguilar, A. Mihara and A. A. Natale, Int. J. Mod. Phys. A 19 (2004) 249.

[55] J. S. Ball and T. W. Chiu, Phys. Rev. D 22, 2550 (1980) [Erratum-ibid. D 23, 3085 (1981)].

[56] J. E. King, Phys. Rev. D 27, 1821 (1983).

[57] A. Kizilersu and M. R. Pennington, Phys. Rev. D 79, 125020 (2009).

[58] M. Binger and S. J. Brodsky, Phys. Rev. D 74, 054016 (2006).

[59] J. M. Cornwall and W. S. Hou, Phys. Rev. D 34, 585 (1986).

[60] For a detailed discussion of the positivity issues related to the spectral representation of the PT gluon propagator and their resolution, see [52].

[61] In the derivation of the second identity one assumes that $\int d \lambda^{2} \rho\left(\lambda^{2}\right)=0$, see [1]. 